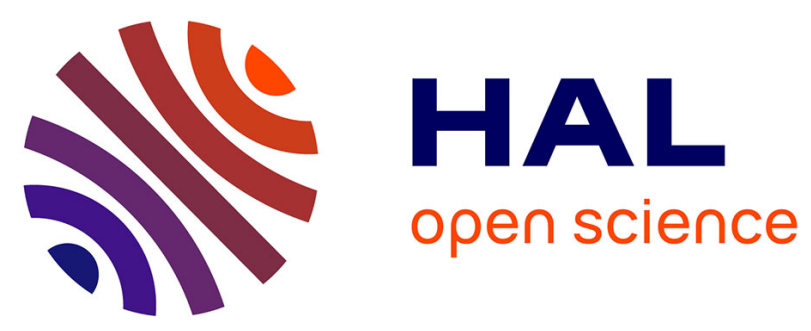

\title{
Computing Distributed Knowledge as the Greatest Lower Bound of Knowledge
}

Carlos Pinzón, Santiago Quintero, Sergio Ramírez, Frank D. Valencia

\section{To cite this version:}

Carlos Pinzón, Santiago Quintero, Sergio Ramírez, Frank D. Valencia. Computing Distributed Knowledge as the Greatest Lower Bound of Knowledge. RAMICS 2021 - Relational and Algebraic Methods in Computer Science, Nov 2021, Marseille, France. pp.413-432. hal-02422624v2

\section{HAL Id: hal-02422624 \\ https://hal.science/hal-02422624v2}

Submitted on 21 Jan 2020

HAL is a multi-disciplinary open access archive for the deposit and dissemination of scientific research documents, whether they are published or not. The documents may come from teaching and research institutions in France or abroad, or from public or private research centers.
L'archive ouverte pluridisciplinaire HAL, est destinée au dépôt et à la diffusion de documents scientifiques de niveau recherche, publiés ou non, émanant des établissements d'enseignement et de recherche français ou étrangers, des laboratoires publics ou privés. 


\title{
Counting and Computing Join-Endomorphisms in Lattices *
}

\author{
Santiago Quintero ${ }^{2}$, Sergio Ramirez ${ }^{1}$, Camilo Rueda $^{1}$, Frank Valencia ${ }^{1,3}$ \\ 1 Pontificia Universidad Javeriana Cali \\ 2 LIX, École Polytechnique de Paris \\ 3 CNRS-LIX, École Polytechnique de Paris
}

\begin{abstract}
Structures involving a lattice and join-endomorphisms on it are ubiquitous in computer science. We study the cardinality of the set $\mathcal{E}(L)$ of all joinendomorphisms of a given finite lattice $L$. In particular, we show that when $L$ is $\mathbf{M}_{n}$, the discrete order of $n$ elements extended with top and bottom, $|\mathcal{E}(L)|=$ $n ! \mathcal{L}_{n}(-1)+(n+1)^{2}$ where $\mathcal{L}_{n}(x)$ is the Laguerre polynomial of degree $n$. We also study the following problem: Given a lattice $L$ of size $n$ and a set $S \subseteq$ $\mathcal{E}(L)$ of size $m$, find the greatest lower bound $\prod_{\mathcal{E}(L)} S$. The join-endomorphism $\prod_{\mathcal{E}(L)} S$ has meaningful interpretations in epistemic logic, distributed systems, and Aumann structures. We show that this problem can be solved with worst-case time complexity in $O(n+m \log n)$ for powerset lattices, $O\left(m n^{2}\right)$ for lattices of sets, and $O\left(m n+n^{3}\right)$ for arbitrary lattices. The complexity is expressed in terms of the basic binary lattice operations performed by the algorithm.
\end{abstract}

\section{Introduction}

There is a long established tradition of using lattices to model structural entities in many fields of mathematics and computer science. For example, lattices are used in concurrency theory to represent the hierarchical organization of the information resulting from agent's interactions [13]. Mathematical morphology (MM), a well-established theory for the analysis and processing of geometrical structures, is founded upon lattice theory [214]. Lattices are also used as algebraic structures for modal and epistemic logics as well as Aumann structures (e.g., modal algebras and constraint systems [8]).

In all these and many other applications, lattice join-endomorphisms appear as fundamental. In MM, join-endomorphisms correspond to one of its fundamental operations; dilations. In modal algebra, they correspond via duality to the box modal operator. In epistemic settings, they represent belief or knowledge of agents. In fact, our own interest in lattice theory derives from using join-endomorphisms to model the perception that agents may have of a statement in a lattice of partial information [8].

For finite lattices, devising suitable algorithms to compute lattice maps with some given properties would thus be of great utility. We are interested in constructing algorithms for computing lattice morphisms. This requires, first, a careful study of the space of such maps to have a clear idea of how particular lattice structures impact on

${ }^{\star}$ This work has been partially supported by the ECOS-NORD project FACTS (C19M03) 
the size of the space. We are, moreover, particularly interested in computing the maximum join-endomorphism below a given collection of join-morphisms. This turns out to be important, among others, in spatial computation (and in epistemic logic) to model the distributed information (resp. distributed knowledge) available to a set of agents as conforming a group [9]. It could also be regarded as the maximum perception consistent with (or derivable from) a collection of perceptions of a group of agents.

Problem. Consider the set $\mathcal{E}(L)$ of all join-endomorphisms of a finite lattice $L$. The set $\mathcal{E}(L)$ can be made into a lattice by ordering join-endomorphisms point-wise wrt the order of $L$. We investigate the following maximization problem: Given a lattice $L$ of size $n$ and a set $S \subseteq \mathcal{E}(L)$ of size $m$, find in $\mathcal{E}(L)$ the greatest lower bound of $S$, i.e., $\prod_{\mathcal{E}(L)} S$. Simply taking $\sigma: L \rightarrow L$ with $\sigma(e) \stackrel{\text { def }}{=} \prod_{L}\{f(e) \mid f \in S\}$ does not solve the problem as $\sigma$ may not be a join-endomorphism. Furthermore, since $\mathcal{E}(L)$ can be seen as the search space, we also consider the problem of determining its cardinality. Our main results are the following.

This paper. We present characterizations of the exact cardinality of $\mathcal{E}(L)$ for some fundamental lattices. Our contribution is to establish the cardinality of $\mathcal{E}(L)$ for the stereotypical non-distributive lattice $L=\mathbf{M}_{n}$. We show that $\left|\mathcal{E}\left(\mathbf{M}_{n}\right)\right|$ equals $r_{0}^{n}+\ldots+$ $r_{n}^{n}+r_{1}^{n+1}=n ! \mathcal{L}_{n}(-1)+(n+1)^{2}$ where $r_{k}^{m}$ is the number of ways to place $k$ nonattacking rooks on an $m \times m$ board and $\mathcal{L}_{n}(x)$ is the Laguerre polynomial of degree $n$. We also present cardinality results for powerset and linear lattices that are part of the lattice theory folklore: The number of join-endomorphisms is $n^{\log _{2} n}$ for powerset lattices of size $n$ and $\left(\begin{array}{c}2 n \\ n\end{array}\right)$ for linear lattices of size $n+1$. Furthermore, we provide algorithms that, given a lattice $L$ of size $n$ and a set $S \subseteq \mathcal{E}(L)$ of size $m$, compute $\prod_{\mathcal{E}(L)} S$. Our contribution is to show that $\prod_{\mathcal{E}(L)} S$ can be computed with worst-case time complexity in $O(n+m \log n)$ for powerset lattices, $O\left(m n^{2}\right)$ for lattices of sets, and $O\left(n m+n^{3}\right)$ for arbitrary lattices.

\section{Background: Join-Endomorphisms and Their Space}

We presuppose basic knowledge of order theory [3] and use the following notions. Let ( $L, \sqsubseteq$ ) be a partially ordered set (poset), and let $S \subseteq L$. We use $\bigsqcup_{L} S$ to denote the least upper bound (or supremum or join) of $S$ in $L$, if it exists. Dually, $\prod_{L} S$ is the greatest lower bound (glb) (infimum or meet) of $S$ in $L$, if it exists. We shall often omit the index $L$ from $\bigsqcup_{L}$ and $\prod_{L}$ when no confusion arises. As usual, if $S=\{c, d\}, c \sqcup d$ and $c \sqcap d$ represent $\bigsqcup S$ and $\Pi S$, respectively. If $L$ has a greatest element (top) $\top$, and a least element (bottom) $\perp$, we have $\bigsqcup \emptyset=\perp$ and $\sqcap \emptyset=\top$. The poset $L$ is distributive iff for every $a, b, c \in L, a \sqcup(b \sqcap c)=(a \sqcup b) \sqcap(a \sqcup c)$.

The poset $L$ is a lattice iff each finite nonempty subset of $L$ has a supremum and infimum in $L$, and it is a complete lattice iff each subset of $L$ has a supremum and infimum in $L$. A self-map on $L$ is a function $f: L \rightarrow L$. A self-map $f$ is monotonic if $a \sqsubseteq b$ implies $f(a) \sqsubseteq f(b)$. We say that $f$ preserves the join of $S \subseteq L$ iff $f(\bigsqcup S)=$ $\bigsqcup\{f(c) \mid c \in S\}$.

We shall use the following posets and notation. Given $n$, we use $\mathbf{n}$ to denote the poset $\{1, \ldots, n\}$ with the linear order $x \sqsubseteq y$ iff $x \leq y$. The poset $\overline{\mathbf{n}}$ is the set $\{1, \ldots, n\}$ with the discrete order $x \sqsubseteq y$ iff $x=y$. Given a poset $L$, we use $L_{\perp}$ for the poset that 
results from adding a bottom element to $L$. The poset $L^{\top}$ is similarly defined. The lattice $\mathbf{2}^{n}$ is the $n$-fold Cartesian product of $\mathbf{2}$ ordered coordinate-wise. We define $\mathbf{M}_{n}$ as the lattice $\left(\overline{\mathbf{n}}_{\perp}\right)^{\top}$. A lattice of sets is a set of sets ordered by inclusion and closed under finite unions and intersections. A powerset lattice is a lattice of sets that includes all the subsets of its top element.

We shall investigate the set of all join-endomorphisms of a given lattice ordered point-wise. Notice that every finite lattice is a complete lattice.

Definition 1 (Join-endomorphisms and their space). Let $L$ be a complete lattice. We say that a self-map is a (lattice) join-endomorphism iff it preserves the join of every finite subset of $L$. Define $\mathcal{E}(L)$ as the set of all join-endomorphisms of L. Furthermore, given $f, g \in \mathcal{E}(L)$, define $f \sqsubseteq \mathcal{E} g$ iff $f(a) \sqsubseteq g(a)$ for every $a \in L$.

The following are immediate consequences of the above definition.

Proposition 1. Let $L$ be a complete lattice. $f \in \mathcal{E}(L)$ iff $f(\perp)=\perp$ and $f(a \sqcup b)=$ $f(a) \sqcup f(b)$ for all $a, b \in L$. If $f$ is a join-endomorphism of $L$ then $f$ is monotonic.

Given a set $S \subseteq \mathcal{E}(L)$, where $L$ is a finite lattice, we are interested in finding the greatest join-endomorphism in $\mathcal{E}(L)$ below the elements of $S$, i.e., $\prod_{\mathcal{E}(L)} S$. Since every finite lattice is also a complete lattice, the existence of $\prod_{\mathcal{E}(L)} S$ is guaranteed by the following proposition.

Proposition 2 ([7]). If $(L, \sqsubseteq)$ is a complete lattice, $(\mathcal{E}(L), \sqsubseteq \mathcal{E})$ is a complete lattice.

In the following sections we study the cardinality of $\mathcal{E}(L)$ for some fundamental lattices and provide efficient algorithms to compute $\prod_{\mathcal{E}(L)} S$.

\section{The Size of the Function Space}

The main result of this section is Theorem 1. It states the size of $\mathcal{E}(L)$ when $L$ is a discrete order extended with a top and bottom. Propositions 3 and 4 state, respectively, the size of $\mathcal{E}(L)$ for the cases when $L$ is a powerset lattice and when $L$ is a total order.

\subsection{Distributive Lattices}

We begin with lattices isomorphic to $\mathbf{2}^{n}$. They include finite boolean algebras and powerset lattices [3]. The size of these lattices are easy to determine from the observation that their join-preserving are determined by their action on their atoms.

Proposition 3. Suppose that $m \geq 0$. Let $L$ be any lattice isomorphic to the product lattice $2^{m}$. Then $|\mathcal{E}(L)|=n^{\log _{2} n}$ where $n=2^{m}$ is the size of $L$.

Thus powerset lattices and boolean algebras have a super-polynomial, sub-exponential number of join-endomorphisms. Nevertheless, linear order lattices allow for an exponential number of join-endomorphisms given by the central binomial coefficient. The following proposition is also easy to prove from the observation that the joinhomorphisms over a linear order are also monotonic functions. In fact, this result appears in [1] and it is well-known among RAMICS community [11|16]. 
Proposition 4. Suppose that $n \geq 0$. Let $L$ be any lattice isomorphic to the linear order lattice $\mathbf{n}_{\perp}$. Then $|\mathcal{E}(L)|=\left(\begin{array}{c}2 n \\ n\end{array}\right)$.

It is easy to prove that $\frac{4^{n}}{2 \sqrt{n}} \leq\left(\begin{array}{c}2 n \\ n\end{array}\right) \leq 4^{n}$ for $n \geq 1$. Together with Prop 4 this gives us explicit exponential lower and upper bounds for $|\mathcal{E}(L)|$ when $L$ is a linear lattice.

\subsection{Non-distributive Case}

The number of join-endomorphisms for some non-distributive lattices of a given size can be much bigger than that for those distributive lattice of the same size in the previous section. We will characterize this number for an archetypal non-distributive lattice in terms of Laguerre (and rook) polynomials.

Laguerre polynomials are solutions to Laguerre's second-order linear differential equation $x y^{\prime \prime}+(1-x) y^{\prime}+n y=0$ where $y^{\prime}$ and $y^{\prime \prime}$ are the first and second derivatives of an unknown function $y$ of the variable $x$ and $n$ is a non-negative integer. The Laguerre polynomial of degree $n$ in $x, \mathcal{L}_{n}(x)$ is given by the summation $\sum_{k=0}^{n}\left(\begin{array}{l}n \\ k\end{array}\right) \frac{(-1)^{k}}{k !} x^{k}$.

The lattice $\mathbf{M}_{n}$ is non-distributive for any $n \geq 3$. The size of $\mathcal{E}\left(\mathbf{M}_{n}\right)$ can be succinctly expressed as follows.

Theorem 1. $\left|\mathcal{E}\left(\mathbf{M}_{n}\right)\right|=(n+1)^{2}+n ! \mathcal{L}_{n}(-1)$.

In combinatorics rook polynomials are generating functions of the number of ways to place non-attacking rooks on a board. A rook polynomial (for square boards) $\mathcal{R}_{n}(x)$ has the form $\sum_{k=0}^{n} x^{k} r(k, n)$ where the (rook) coefficient $r(k, n)$ represents the number of ways to place $k$ non-attacking rooks on an $n \times n$ chessboard. For instance, $r(0, n)=1, r(1, n)=n^{2}$ and $r(n, n)=n !$. In general $r(k, n)=\left(\begin{array}{l}n \\ k\end{array}\right)^{2} k !$.

Rook polynomials are related to Laguerre polynomials by $\mathcal{R}_{n}(x)=n ! x^{n} \mathcal{L}_{n}\left(-x^{-1}\right)$. Therefore, as a direct consequence of the above theorem, we can also characterize $\left|\mathcal{E}\left(\mathbf{M}_{n}\right)\right|$ in combinatorial terms as the following sum of rook coefficients.

Corollary 1. Let $r^{\prime}(n+1, n)=r(1, n+1)$ and $r^{\prime}(k, n)=r(k, n)$ if $k \leq n$. Then $\left|\mathcal{E}\left(\mathbf{M}_{n}\right)\right|=\sum_{k=0}^{n+1} r^{\prime}(k, n)$.

We conclude this section with another pleasant correspondence between the endomorphisms in $\mathcal{E}\left(\mathbf{M}_{n}\right)$ and $\mathcal{R}_{n}(x)$. Let $f: L \rightarrow L$ be a function over a lattice $(L, \sqsubseteq)$. We say that $f$ is non-reducing in $L$ iff it does not map any value to a smaller one; i.e., there is no $e \in L$ such that $f(e) \sqsubset e$. The number of join-endomorphisms that are non-reducing in $\mathbf{M}_{n}$ is exactly the value of the rook polynomial $\mathcal{R}_{n}(x)$ for $x=1$.

Corollary 2. $\mathcal{R}_{n}(1)=\mid\left\{f \in \mathcal{E}\left(\mathbf{M}_{n}\right) \mid f\right.$ is non-reducing in $\left.\mathbf{M}_{n}\right\} \mid$.

Table 11 illustrates the join-endomorphisms over the lattice $\mathbf{M}_{n}$ as the union of four families $\mathcal{F}_{1}, \ldots, \mathcal{F}_{4}$. Corollary 2 follows from the observation that the set of nonreducing functions in $\mathbf{M}_{n}$ is equal to $\mathcal{F}_{4}$ whose size is $\mathcal{R}_{n}(1)$ as shown in the following proof of Th. 1 . 


\subsection{Proofs}

We present proofs for the statements of this section. Propositions 3 and 4 follow from simple observations and they are part of the lattice theory folklore [1111|16]. We present original proofs of these proposition in the Appendix.

Proof of Proposition 3 . We wish to prove the following: Let $L$ be any lattice isomorphic to the product lattice $\mathbf{2}^{m}$. Then $|\mathcal{E}(L)|=n^{\log _{2} n}$ where $n=2^{m}$ is the size of $L$.

Take any set $S$ of size $m$. Consider the powerset lattice $L=\mathcal{P}(S)$ ordered by inclusion. We have $n=|\mathcal{P}(S)|=2^{m}$. We shall show that $|\mathcal{E}(L)|=n^{\log _{2} n}$. Since $\mathcal{P}(S)$ is isomorphic to $\mathbf{2}^{m}$, Proposition 3 follows from the fact that isomorphic lattices have the same number of join-endomorphisms.

Let $\mathcal{F}$ be the family of functions $f: \mathcal{P}(S) \rightarrow \mathcal{P}(S)$ that satisfy (a) $f(T)=$ $\bigcup_{t \in T} f(\{t\})$ if $|T|>1$ and (b) $f(\emptyset)=\emptyset$. The equality $|\mathcal{E}(L)|=n^{\log _{2} n}$ follows from the following claim: (1) $\mathcal{F}=\mathcal{E}(L)$ and (2) $|\mathcal{F}|=n^{\log _{2} n}$.

To prove (1) one can verify that if $f \in \mathcal{F}$ then $f$ is a join-endomorphism where $\sqcup$ is $\cup$ and $\perp$ is the $\emptyset$. Hence $f \in \mathcal{E}(L)$. On the other hand, if $f \notin \mathcal{F}$ then either $f(T) \neq \bigcup_{t \in T} f(\{t\})$ for some $T \subseteq S$ or $f(\emptyset) \neq \emptyset$. But since $\sqcup=\cup$ and $\perp=\emptyset$, we have $T=\bigsqcup_{t \in T}\{t\}$ but $f(T) \neq \bigsqcup_{t \in T} f(\{t\})$ or $f(\perp) \neq \perp$. Hence $f \notin \mathcal{E}(L)$.

To prove (2) notice that given $f \in \mathcal{F}$, for each $T \subseteq S$ if $|T|>1$ then the value $f(T)$ is determined by the values of $f$ applied to each singleton $\{t\} \subseteq S$, and if $|T|=0$ the value $f(T)$ is fixed to $\emptyset$. The set $\mathcal{P}(S)$ has $\log _{2} n=m$ singletons. Since there is no restriction on how each $f \in \mathcal{F}$ should map singletons, $|\mathcal{F}|=n^{\log _{2} n}$ as wanted.

Proof of Proposition 4. We now show that the size of $\mathcal{E}(L)$ for linear orders is determined by the central binomial coefficient. Let $L$ be any lattice isomorphic to the linear order lattice $\mathbf{n}_{\perp}$. We want to show that $|\mathcal{E}(L)|=\left(\begin{array}{c}2 n \\ n\end{array}\right)$.

Let $\mathcal{M}_{\perp}(L)$ be the set of monotonic functions from $L$ to $L$ that preserve $\perp$. We claim that $\mathcal{M}_{\perp}(L)=\mathcal{E}(L)$. The inclusion $\mathcal{E}(L) \subseteq \mathcal{M}_{\perp}(L)$ follows from Proposition 1 and the fact that join-endomorphisms preserve bottoms. For $\mathcal{M}_{\perp}(L) \subseteq \mathcal{E}(L)$, take $f \in \mathcal{M}_{\perp}(L)$. By definition $f(\perp)=\perp$. Take any $a, b \in L$. So either $a \sqsubseteq b$ or $b \sqsubseteq a$. If $a \sqsubseteq b$ then $f(a \sqcup b)=f(b)$ and by monotonicity of $f, f(b)=f(a) \sqcup f(b)$. Similarly if $b \sqsubseteq a$ then $f(a \sqcup b)=f(a)=f(a) \sqcup f(b)$. We conclude that $f \in \mathcal{E}(L)$.

Now, for every $f \in \mathcal{M}_{\perp}(L)$ we have $f(\perp)=\perp$, then $|\mathcal{E}(L)|=\left|\mathcal{M}_{\perp}(L)\right|=$ $|\mathcal{M}(L \backslash\{\perp\} \rightarrow L)|$ where $\mathcal{M}(L \backslash\{\perp\} \rightarrow L)$ is the set of monotonic functions from $L \backslash\{\perp\}$ to $L$. Thus to prove Proposition 4 it suffices to show $|\mathcal{M}(L \backslash\{\perp\} \rightarrow L)|=\left(\begin{array}{c}2 n \\ n\end{array}\right)$.

Notice that $|L|=n+1$. Consider the equation $\sum_{i=1}^{n+1} X_{i}=n$ where the variable $X_{i}$ takes a value between 0 and $n$. Let $\operatorname{Sol}(n)$ be the set of all solutions to this equation. We can show that $|\operatorname{Sol}(n)|=|\mathcal{M}(L \backslash\{\perp\} \rightarrow L)|$ by providing the following bijection $\sigma: \mathcal{M}(L \backslash\{\perp\} \rightarrow L) \rightarrow \operatorname{Sol}(n)$. The function $\sigma$ associates each $f \in \mathcal{M}(L \backslash\{\perp\} \rightarrow$ $L)$ with a solution $\sigma(f)$ assigning to every $X_{i}$ the number of consecutive values from $L \backslash\{\perp\}$ mapped by $f$ to the $i$-th value of $L$.

From combinatorics we know that for any pair of positive integers $n$ and $k$, the number of $k$-tuples of non-negative integers whose sum is $n$ equals $\left(\begin{array}{c}n+k-1 \\ n\end{array}\right)$ [5]. For 

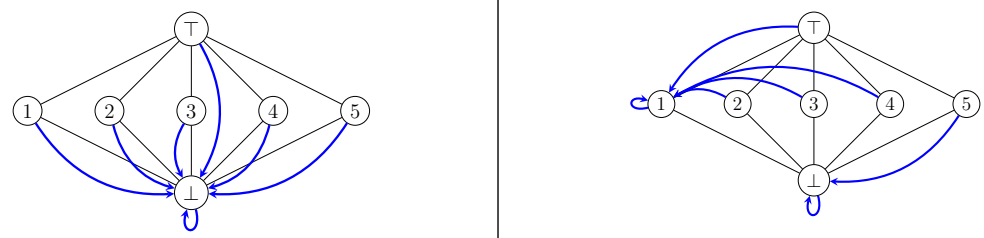

Let $\mathcal{F}_{1}$ be the family of functions $f$ that for all Let $\mathcal{F}_{2}$ be the family of bottom preserving func$e \in \mathbf{M}_{n}, f(e)=\perp$.

tions $f$ such that for some $e, e^{\prime} \in I$ : (a) $f(\top)=$ $e$, (b) $f\left(e^{\prime}\right)=\perp$ or $f\left(e^{\prime}\right)=e$, and (c) $f\left(e^{\prime \prime}\right)=$ $e$ for all $e^{\prime \prime} \in I \backslash\left\{e^{\prime}\right\}$.
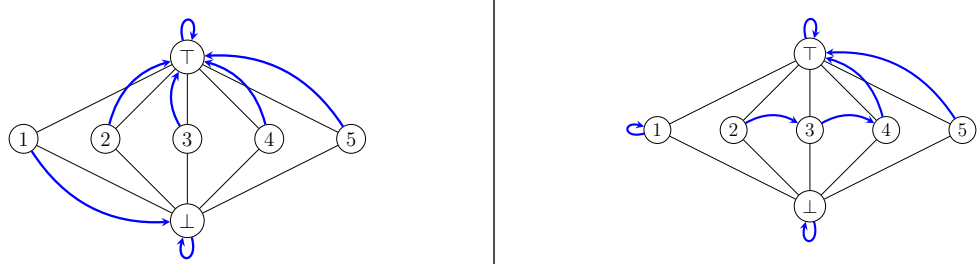

Let $\mathcal{F}_{3}$ be the family of top and bottom pre- Let $\mathcal{F}_{4}$ be the family of top and bottom preservserving functions $f$ such that for some $e \in I$ : ing functions $f$ that for some $J \subseteq I$ :

(a) $f(e)=\perp$, and (b) $f\left(e^{\prime}\right)=\top$ for all (a) $f(e)=\top$ for every $e \in J$, (b) $f \uparrow_{I \backslash J}$ is $e^{\prime} \in I \backslash\{e\}$. injective, and (c) $\operatorname{Img}\left(f \uparrow_{I \backslash J}\right) \subseteq I$.

Table 1: Families $\mathcal{F}_{1}, \ldots, \mathcal{F}_{4}$ of join-endomorphisms of $\mathbf{M}_{n} . I=\{1, \ldots, n\} . f \uparrow_{A}$ is the restriction of $f$ to a subset $A$ of its domain. $\operatorname{Img}(f)$ is the image of $f$. A function from each $\mathcal{F}_{i}$ for $\mathbf{M}_{5}$ is depicted with blue arrows.

$k=n+1$ these tuples correspond exactly to the solutions in $\operatorname{Sol}(n)$. Therefore we have shown $|\mathcal{E}(L)|=\left|\mathcal{M}_{\perp}(L)\right|=|\mathcal{M}(L \backslash\{\perp\} \rightarrow L)|=|\operatorname{Sol}(n)|=\left(\begin{array}{c}2 n \\ n\end{array}\right)$ as wanted.

Proof of Theorem 1. We shall show that $\left|\mathcal{E}\left(\mathbf{M}_{n}\right)\right|$ can be expressed in terms of Laguerre polynomials: $\left|\mathcal{E}\left(\mathbf{M}_{n}\right)\right|=(n+1)^{2}+n ! \mathcal{L}_{n}(-1)$.

Let $\mathcal{F}=\bigcup_{i=1}^{4} \mathcal{F}_{i}$ where the mutually exclusive $\mathcal{F}_{i}$ 's are defined in Table 1 , and $I=\{1, \ldots, n\}$. The proof is divided in two parts: (I) $\mathcal{F}=\mathcal{E}\left(\mathbf{M}_{n}\right)$ and (II) $|\mathcal{F}|=$ $(n+1)^{2}+n ! \mathcal{L}_{n}(-1)$.

Part (I) For $\mathcal{F} \subseteq \mathcal{E}\left(\mathbf{M}_{n}\right)$, it is easy to verify that each $f \in \mathcal{F}$ is a join-endomorphism. For $\mathcal{E}\left(\mathbf{M}_{n}\right) \subseteq \mathcal{F}$ we show that for any function $f$ from $\mathbf{M}_{n}$ to $\mathbf{M}_{n}$ if $f \notin \mathcal{F}$, then $f \notin \mathcal{E}\left(\mathbf{M}_{n}\right)$. Immediately, if $f(\perp) \neq \perp$ then $f \notin \mathcal{E}\left(\mathbf{M}_{n}\right)$.

Suppose $f(\perp)=\perp$. Let $J, K, H$ be disjoint possibly empty sets such that $I=$ $J \cup K \cup H$ and let $j=|J|, k=|K|$ and $h=|H|$. The sets $J, K, H$ represent the elements of $I$ mapped by $f$ to $\top$, to elements of $I$, and to $\perp$, respectively. More 
precisely, $\operatorname{Img}\left(f \uparrow_{J}\right)=\{\top\}, \operatorname{Img}\left(f \uparrow_{K}\right) \subseteq I$ and $\operatorname{Img}\left(f \uparrow_{H}\right)=\{\perp\}$. Furthermore, for every $f$ either (1) $f(\top)=\perp$, (2) $f(T) \in I$ or (3) $f(\top)=\top$. We show that $f \notin \mathcal{E}\left(\mathbf{M}_{n}\right)$.

1. Case $f(\top)=\perp$.

Since $f \notin \mathcal{F}_{1}$ there is an $e \in I$ such that $f(e) \neq \perp$. We have $e \sqsubseteq \top$ but $f(e) \nsubseteq$ $f(\top)$. Then $f$ is not monotonic. From Prop. 1 we conclude $f \notin \mathcal{E}\left(\mathbf{M}_{n}\right)$.

2. Case $f(T) \in I$.

Let $K_{1}, K_{2}$ be disjoint possibly empty sets such that $K_{1} \cup K_{2}=K, \operatorname{Img}\left(f \uparrow_{K_{1}}\right)=$ $\{f(\top)\}$ and $\operatorname{Img}\left(\left.f\right|_{K_{2}}\right) \neq\{f(\top)\}$. Notice that if $j>0$ or $\left|K_{2}\right|>0, f$ is nonmonotonic and then $f \notin \mathcal{E}\left(\mathbf{M}_{n}\right)$.

Now, for $j=0$ and $K_{2}=\emptyset$. Since $\operatorname{Img}\left(f \uparrow_{K}\right)=\{f(\top)\}$ and $f \notin \mathcal{F}_{2}$ then $h>1$. Therefore there must be $e_{1}, e_{2} \in H$ such that $f\left(e_{1}\right)=f\left(e_{2}\right)=\perp$. This implies $f\left(e_{1} \sqcup e_{2}\right)=f(\top) \neq \perp=f\left(e_{1}\right) \sqcup f\left(e_{2}\right)$, therefore $f \notin \mathcal{E}\left(\mathbf{M}_{n}\right)$.

3. Case $f(T)=T$.

3.1. Suppose $k=0$. Notice that $f \notin \mathcal{F}_{3}$ and $f \notin \mathcal{F}_{4}$ hence $h \neq 1$ and $h \neq 0$. Thus $h>1$ implies that there are at least two $e_{1}, e_{2} \in H$ such that $f\left(e_{1}\right)=$ $f\left(e_{2}\right)=\perp$. But then $f\left(e_{1} \sqcup e_{2}\right)=f(\top)=\top \neq \perp=f\left(e_{1}\right) \sqcup f\left(e_{2}\right)$, hence $f \notin \mathcal{E}\left(\mathbf{M}_{n}\right)$.

3.2. Suppose $k>0$. Assume $h=0$. Notice that $K=I \backslash J$ and $\operatorname{Img}\left(f \uparrow_{K}\right) \subseteq I$. Since $f$ is a $\perp$ and $T$ preserving function and it satisfies conditions (a) and (c) of $\mathcal{F}_{4}$ but $f \notin \mathcal{F}_{4}$, then $f$ must violate condition (b). Thus $f \uparrow_{K}$ is not injective. Then there are $a, b \in K$ such that $a \neq b$ but $f(a)=f(b)$. Then $f(a) \sqcup f(b) \neq \top=f(a \sqcup b)$. Consequently, $f \notin \mathcal{E}\left(\mathbf{M}_{n}\right)$.

Assume $h>0$. There must be $e_{1}, e_{2}, e_{3} \in I$ such that $f\left(e_{1}\right)=\perp$ and $f\left(e_{2}\right)=$ $e_{3}$. Notice that $f\left(e_{1}\right) \sqcup f\left(e_{2}\right)=e_{3} \neq \top=f(\top)=f\left(e_{1} \sqcup e_{2}\right)$. Therefore, $f \notin \mathcal{E}\left(\mathbf{M}_{n}\right)$.

Part (II) We prove that $|\mathcal{F}|=\sum_{i=1}^{4}\left|\mathcal{F}_{i}\right|=(n+1)^{2}+n ! \mathcal{L}_{n}(-1)$. Recall that $n=|I|$. It is easy to prove that $\left|\mathcal{F}_{1}\right|=1,\left|\mathcal{F}_{2}\right|=n^{2}+n$ and $\left|\mathcal{F}_{3}\right|=n$.

1. $\left|\mathcal{F}_{1}\right|=1$.

There is only one function mapping every element in $\mathbf{M}_{n}$ to $\perp$.

2. $\left|\mathcal{F}_{2}\right|=n^{2}+n$.

Since $T$ is mapped to an element of $I$, there are $n$ possibilities to choose such element. If there is an element of $I$ mapped to $\perp$, for each one of the previous $n$ options there are also $n$ possibilities to choose an element of $I$ to be mapped to $\perp$. Then, in this case there are $n^{2}$ functions. If no element of $I$ is mapped to $\perp$, then there are $n$ additional functions.

3. $\left|\mathcal{F}_{3}\right|=n$.

One of the elements of $I$ is mapped to $\perp$. All the other elements of $I$ are mapped to $T$. Then, there are $n$ functions that can be defined in $\mathcal{F}_{3}$.

4. $\left|\mathcal{F}_{4}\right|=n ! \mathcal{L}_{n}(-1)$.

Let $f \in \mathcal{F}_{4}$ and let $J \subseteq I$ be a possibly empty set such that $\operatorname{Img}\left(f \uparrow_{J}\right)=\{\top\}$ and $\operatorname{Img}\left(f \uparrow_{I \backslash J}\right) \subseteq I$, where $f \uparrow_{I \backslash J}$ is an injective function. We shall call $j=|J|$.

For each of the $\left(\begin{array}{l}n \\ j\end{array}\right)$ possibilities for $J$, the elements of $I \backslash J$ are to be mapped to $I$ by the injective function $f \uparrow_{I \backslash J}$. The number of functions $f \uparrow_{I \backslash J}$ is $\frac{n !}{j !}$. Therefore, 


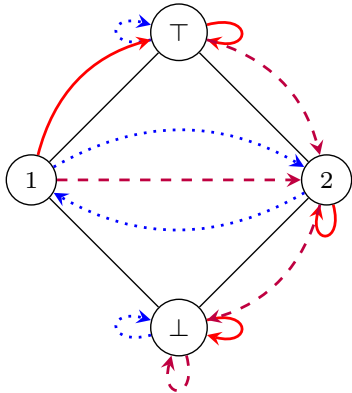

(a) $f_{1}: \cdots \rightarrow, f_{2}: \rightarrow, f:-\rightarrow$

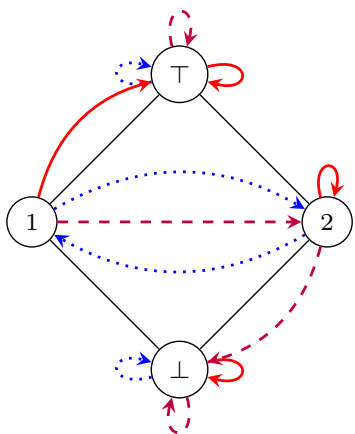

(b) $f_{1}: \cdots \rightarrow, f_{2}: \rightarrow, \sigma_{S}:--\rightarrow$

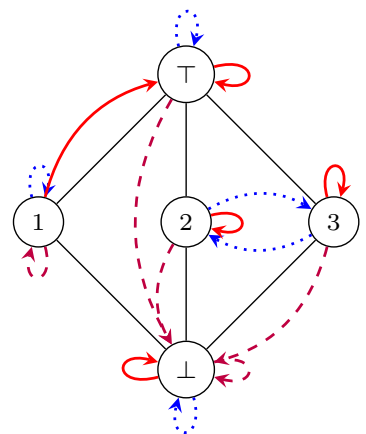

(c) $f_{1}: \cdots \rightarrow, f_{2}: \rightarrow, \delta_{S}:-\rightarrow$

Fig. 1: $S=\left\{f_{1}, f_{2}\right\} \subseteq \mathcal{E}(L)$. (a) $f=\prod_{\mathcal{E}(L)} S$. (b) $\sigma_{S}(c) \stackrel{\text { def }}{=} f_{1}(c) \sqcap f_{2}(c)$ is not a joinendomorphism of $\mathbf{M}_{2}: \sigma_{S}(1 \sqcup 2) \neq \sigma_{S}(1) \sqcup \sigma_{S}(2)$. (c) $\delta_{S}$ in Lemma 1 is not a joinendomorphism of the non-distributive lattice $\mathbf{M}_{3}: \delta_{S}(1) \sqcup \delta_{S}(2)=1 \neq \perp=\delta_{S}(1 \sqcup 2)$.

$\left|\mathcal{F}_{4}\right|=\sum_{j=0}^{n}\left(\begin{array}{c}n \\ j\end{array}\right) \frac{n !}{j !}$. This sum equals $n ! \mathcal{L}_{n}(-1)$ which in turn is equal to $\mathcal{R}_{n}(1)$.

It follows that $|\mathcal{F}|=\sum_{i=1}^{4}\left|\mathcal{F}_{i}\right|=(n+1)^{2}+n ! \mathcal{L}_{n}(-1)$ as wanted.

It follows that $|\mathcal{F}|=\sum_{i=1}^{4}\left|\mathcal{F}_{i}\right|=(n+1)^{2}+n ! \mathcal{L}_{n}(-1)$, as wanted.

\section{Algorithms}

We shall provide efficient algorithms for the maximization problem mentioned in the introduction: Given $L$ and $S \subseteq \mathcal{E}(L)$ find $\prod_{\mathcal{E}(L)} S$, i.e., the greatest join-endomorphism in the lattice $\mathcal{E}(L)$ below all the elements of $S$.

Finding $\prod_{\mathcal{E}(L)} S$ may not be immediate. E.g., see $\prod_{\mathcal{E}(L)} S$ in Fig 1 a for a small lattice of four elements and two join-endomorphisms. As already mentioned, a naive approach is to compute $\prod_{\mathcal{E}(L)} S$ by taking $\sigma_{S}(c) \stackrel{\text { def }}{=} \prod_{L}\{f(c) \mid f \in S\}$ for each $c \in L$. This does not work since $\sigma_{S}$ is not necessarily a join-endomorphism as shown in Fig $1 \mathrm{~b}$

A brute force solution to computing $\prod_{\mathcal{E}(L)} S$ can be obtained by generating the set $S^{\prime}=\{g \mid g \in \mathcal{E}(L)$ and $g \sqsubseteq f$ for all $f \in S\}$ and taking its join. This approach works since $\bigsqcup S^{\prime}=\prod_{\mathcal{E}(L)} S$ but as shown in Section 3 , the size of $\mathcal{E}(L)$ can be superpolynomial for distributive lattices and exponential in general.

Nevertheless, one can use lattice properties to compute $\prod_{\mathcal{E}(L)} S$ efficiently. For distributive lattices, we use the inherent compositional nature of $\prod_{\mathcal{E}(L)} S$. For arbitrary lattices, we present an algorithm that uses the function $\sigma_{S}$ in the naive approach to compute $\prod_{\mathcal{E}(L)} S$ by approximating it from above.

We will give the time complexities in terms of the number of basic binary lattice operations (i.e., meets, joins and subtractions) performed during execution. 


\subsection{Meet of Join-Endomorphisms in Distributive Lattices}

Here we shall illustrate some pleasant compositionality properties of the infima of joinendomorphisms that can be used for computing the join-endomorphism $\prod_{\mathcal{E}(L)} S$ in a finite distributive lattice $L$. In what follows we assume $n=|L|$ and $m=|S|$.

We use $X^{J}$ to denote the set of tuples $\left(x_{j}\right)_{j \in J}$ of elements $x_{j} \in X$ for each $j \in J$.

Lemma 1. Let $L$ be a finite distributive lattice and $S=\left\{f_{i}\right\}_{i \in I} \subseteq \mathcal{E}(L)$. Then $\prod_{\mathcal{E}(L)} S=\delta_{S}$ where $\delta_{S}(c) \stackrel{\text { def }}{=} \prod_{L}\left\{\bigsqcup_{i \in I} f_{i}\left(a_{i}\right) \mid\left(a_{i}\right)_{i \in I} \in L^{I}\right.$ and $\left.\bigsqcup_{i \in I} a_{i} \sqsupseteq c\right\}$.

The above lemma basically says that $\left(\prod_{\mathcal{E}(L)} S\right)(c)$ is the greatest element in $L$ below all possible applications of the functions in $S$ to elements whose join is greater or equal to $c$. The proof that $\delta_{S} \sqsupseteq_{\mathcal{E}} \prod_{\mathcal{E}(L)} S$ uses the fact that join-endomorphisms preserve joins. The proof that $\delta_{S} \sqsubseteq \mathcal{E} \prod_{\mathcal{E}(L)} S$ proceeds by showing that $\delta_{S}$ is a lower bound in $\mathcal{E}(L)$ of $S$. Distributivity of the lattice $L$ is crucial for this direction. In fact without it $\prod_{\mathcal{E}(L)} S=\delta_{S}$ does not necessarily hold as shown by the following counter-example.

Example 1. Consider the non-distributive lattice $\mathbf{M}_{3}$ and $S=\left\{f_{1}, f_{2}\right\}$ defined as in Fig $1 \mathrm{c}$ We obtain $\delta_{S}(1 \sqcup 2)=\delta_{S}(\top)=\perp$ and $\delta_{S}(1) \sqcup \delta_{S}(2)=1 \sqcup \perp=1$. Then, $\delta_{S}(1 \sqcup 2) \neq \delta_{S}(1) \sqcup \delta_{S}(2)$, i.e., $\delta_{S}$ is not a join-endomorphism.

Naive Algorithm $A_{1}$. One could use Lemma 1 directly in the obvious way to provide an algorithm for $\prod_{\mathcal{E}(L)} S$ by computing $\delta_{S}$ : i.e., computing the meet of elements of the form $\bigsqcup_{i \in I} f_{i}\left(a_{i}\right)$ for every tuple $\left(a_{i}\right)_{i \in I}$ such that $\bigsqcup_{i \in I} a_{i} \sqsupseteq c$. For each $c \in L, \delta_{S}(c)$ checks $n^{m}$ tuples $\left(a_{i}\right)_{i \in I}$, each one with a cost in $O(m)$. Thus $A_{1}$ can compute $\prod_{\mathcal{E}(L)} S$ by performing $O\left(n \times n^{m} \times m\right)=O\left(m n^{m+1}\right)$ binary lattice operations.

Nevertheless, we can use Lemma 1 to provide a recursive characterization of $\prod_{\mathcal{E}(L)} S$ that can be used in a divide-and-conquer algorithm with lower time complexity.

Proposition 5. Let $L$ be a finite distributive lattice and $S=S_{1} \cup S_{2} \subseteq \mathcal{E}(L)$. Then $\left(\prod_{\mathcal{E}(L)} S\right)(c)=\prod_{L}\left\{\left(\prod_{\mathcal{E}(L)} S_{1}\right)(a) \sqcup\left(\prod_{\mathcal{E}(L)} S_{2}\right)(b) \mid a, b \in L\right.$ and $\left.a \sqcup b \sqsupseteq c\right\}$.

The above proposition bears witness to the compositional nature of $\prod_{\mathcal{E}(L)} S$. It can be proven by replacing $\left(\prod_{\mathcal{E}(L)} S_{1}\right)(a)$ and $\left(\prod_{\mathcal{E}(L)} S_{2}\right)(b)$ by $\delta_{S_{1}}(a)$ and $\delta_{S_{2}}(b)$ using Lemma11 (see Appendix A].

Naive Algorithm $A_{2}$. We can use Prop 5 to compute $\prod_{\mathcal{E}(L)} S$ with the following recursive procedure: Take any partition $\left\{S_{1}, S_{2}\right\}$ of $S$ such that the absolute value of $\left|S_{1}\right|-\left|S_{2}\right|$ is at most 1 . Then compute the meet of all $\left(\prod_{\mathcal{E}(L)} S_{1}\right)(a) \sqcup\left(\prod_{\mathcal{E}(L)} S_{2}\right)(b)$ for every $a, b$ such that $a \sqcup b \sqsupseteq c$. Then given $c \in L$, the time complexity of a naive implementation of this algorithm can be obtained as the solution of the equation $T(m)=n^{2}(1+2 T(m / 2))$ and $T(1)=1$ which is in $O\left(m n^{2 \log _{2} m}\right)$. Therefore, $\prod_{\mathcal{E}(L)} S$ can be computed in $O\left(m n^{1+2 \log _{2} m}\right)$.

The time complexity of the naive algorithm $A_{2}$ is better than that of $A_{1}$. However, by using a simple memoization technique to avoid repeating recursive calls and the following observations one can compute $\prod_{\mathcal{E}(L)} S$ in a much lower time complexity order. 


\subsection{Using Subtraction and Downsets to characterize $\prod_{\mathcal{E}(L)} S$}

In what follows we show that $\prod_{\mathcal{E}(L)} S$ can be computed in $O\left(m n^{2}\right)$ for distributive lattices and, in particular, in $O(n+m \log n)$ for powerset lattices. To achieve this we use the subtraction operator from co-Heyting algebras and the notion of down set.

Subtraction Operator. Notice that in Prop 5 we are considering all pairs $a, b \in L$ such that $a \sqcup b \sqsupseteq c$. However, because of the monotonicity of join-endomorphisms, it suffices to take, for each $a \in L$, just the least $b$ such that $a \sqcup b \sqsupseteq c$. In finite distributive lattices, and more generally in co-Heyting algebras [6], the subtraction operator $c \backslash a$ gives us exactly such a least element. The subtraction operator is uniquely determined by the property (Galois connection) $b \sqsupseteq c \backslash a$ iff $a \sqcup b \sqsupseteq c$ for all $a, b, c \in L$.

Down-sets. Besides using just $c \backslash a$ instead of all $b$ 's such that $a \sqcup b \sqsupseteq c$, we can use a further simplification: Rather than including every $a \in L$, we only need to consider every $a$ in the down-set of $c$. Recall that the down-set of $c$ is defined as $\downarrow c=\{e \in$ $L \mid e \sqsubseteq c\}$. This additional simplification is justified using properties of distributive lattices to show that for any $a^{\prime} \in L$, such that $a^{\prime} \nsubseteq c$, there exists $a \sqsubseteq c$ such that $\left(\prod_{\mathcal{E}(L)} S_{1}\right)(a) \sqcup\left(\prod_{\mathcal{E}(L)} S_{2}\right)(c \backslash a) \sqsubseteq\left(\prod_{\mathcal{E}(L)} S_{1}\right)\left(a^{\prime}\right) \sqcup\left(\prod_{\mathcal{E}(L)} S_{2}\right)\left(c \backslash a^{\prime}\right)$.

The above observations lead us to the following theorem.

Theorem 2. Let $L$ be a finite distributive lattice and $S=S_{1} \cup S_{2} \subseteq \mathcal{E}(L)$. Then $\left(\prod_{\mathcal{E}(L)} S\right)(c)=\prod_{L}\left\{\left(\prod_{\mathcal{E}(L)} S_{1}\right)(a) \sqcup\left(\prod_{\mathcal{E}(L)} S_{2}\right)(c \backslash a) \mid a \in \downarrow c\right\}$.

The above result can be used to derive a simple recursive algorithm that, given a finite distributive lattice $L$ and $S \subseteq \mathcal{E}(L)$, computes $\prod_{\mathcal{E}(L)} S$ in worst-case time complexity $O\left(m n^{2}\right)$ where $m=|S|$ and $n=|L|$. We show this algorithm next.

\subsection{Algorithms for Distributive Lattices}

We first describe the algorithm DMEETAPP that computes the value $\left(\prod_{\mathcal{E}(L)} S\right)(c)$. We then describe the algorithm DMEET that computes the function $\prod_{\mathcal{E}(L)} S$ by calling DMEETAPP in a particular order to avoid repeating computations. To specify the calling order we need the following definition.

Definition 2. A binary partition tree (bpt) of a finite set $S \neq \emptyset$ is a binary tree such that (a) its root is $S$, (b) if $|S|=1$ then its root is a leaf, and (c) if $|S|>1$ it has a left and a right subtree, themselves bpts of $S_{1}$ and $S_{2}$ resp., for a partition $\left\{S_{1}, S_{2}\right\}$ of $S$.

Let $\Delta$ be a bpt of $S$. We use $\Delta\left(S^{\prime}\right)$ for the subtree of $\Delta$ rooted at $S^{\prime} \subseteq S$, if it exists. Clearly, $\Delta=\Delta(S)$. We use the triple $\left\langle S, \Delta_{1}, \Delta_{2}\right\rangle$ for the bpt of $S$ with $\Delta_{1}$ and $\Delta_{2}$ as its left and right subtrees.

The following proposition is an immediate consequence of the previous definition.

Proposition 6. The size (number of nodes) of any bpt of $S$ is $2 m-1$ where $m=|S|$. 
$\operatorname{DMeETAPP}(\Delta, c)$. Let $\Delta=\left\langle S, \Delta_{1}, \Delta_{2}\right\rangle$ be a bpt of $S \subseteq \mathcal{E}(L)$ where $L$ is a distributive lattice. The recursive program $\operatorname{DMeETApp}(\Delta, c)$ defined in $\operatorname{Algorithm} 1$ computes $\left(\prod_{\mathcal{E}(L)} S\right)(c)$. It uses a global lookup table $T$ for storing the results of calls to DMEETAPP. Initially each entry of $T$ stores a null value not included in $L$. Since $S$ is the union of the roots of $\Delta_{1}$ and $\Delta_{2}$, the correctness of $\operatorname{DMEETApP}(\Delta, c)$ follows from $\mathrm{Thm} 2$ Termination follows from the fact that $L$ is finite and the bpts $\Delta_{1}$ and $\Delta_{2}$ in the recursive calls are strictly smaller than $\Delta$.

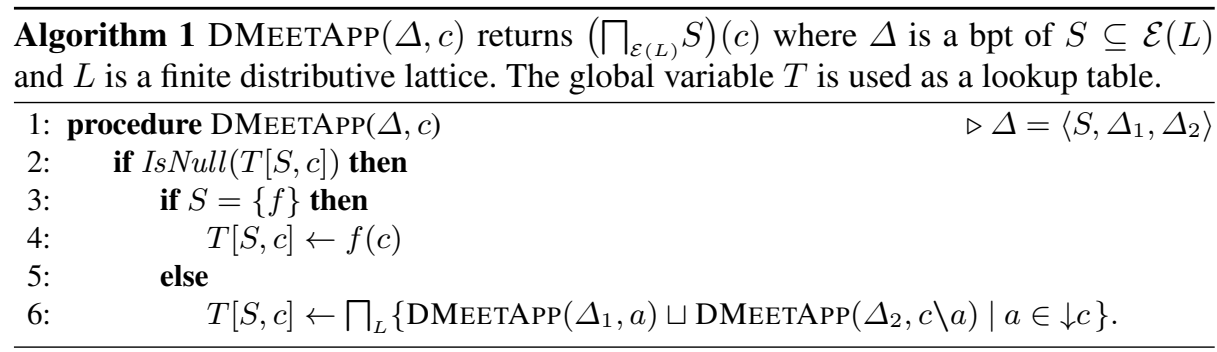

Computing $\prod_{\mathcal{E}(L)} S$ for Distributive Lattices. We show how to compute $\prod_{\mathcal{E}(L)} S$ with a worst-case time complexity in $O\left(m n^{2}\right)$.

Let $L$ be a finite lattice of sets and $\Delta=\left\langle S, \Delta_{1}, \Delta_{2}\right\rangle$ be a bpt of $S \subseteq \mathcal{E}(L)$. Let $n=|L|$ and $m=|S|$. Let us consider an execution of $\operatorname{DMeEtApP}(\Delta, c)$. From the definition of subtraction it follows that $c \backslash a \in \downarrow c$. Then for each recursive call $\operatorname{DMeetApp}\left(\Delta^{\prime}, a^{\prime}\right)$ performed by an execution of DMeetApp $(\Delta, c)$ we have $a^{\prime} \in \downarrow c$.

The above leads us to the following observation about the order of the number of binary lattice operations (meets, joins, and subtractions) performed by $\operatorname{DMeEtApP}(\Delta, c)$.

Observation 3 Let $\Delta=\left\langle S, \Delta_{1}, \Delta_{2}\right\rangle$ with $\Delta_{1}$ and $\Delta_{2}$ rooted at $S_{1}$ and $S_{2}$. Assume that $T\left[S_{1}, a^{\prime}\right], T\left[S_{2}, a^{\prime}\right] \in L$ for every $a^{\prime} \in \downarrow c$. Then the number of binary lattice operations performed by $\operatorname{DMeEtApP}(\Delta, c)$ is in $O(|\downarrow c|)$.

Since each entry of $T$ is initialized with a null value not in $L$, the assumption in Obs 3 implies that for every $a^{\prime} \in \downarrow c$ the values of DMeETApp $\left(\Delta_{1}, a^{\prime}\right)$ and DMEE$\operatorname{TApP}\left(\Delta_{2}, a^{\prime}\right)$ have been previously stored in $T$. Under this condition $\operatorname{DMeETApP}(\Delta, c)$ performs at most $|\downarrow c|$ binary joins, $|\downarrow c|$ subtractions, $|\downarrow c|-1$ binary meets.

$\operatorname{DMeEt}(\boldsymbol{L}, \boldsymbol{S}, \boldsymbol{P})$. The values of $\left(\Pi_{\mathcal{E}(L)} S\right)(c)$ for each $c \in P \subseteq L$ are computed by the program in Algorithm 2 as follows. The program first initializes the table $T$ with a null value. Then, to satisfy the assumption in Obs 3 it traverses $\Delta$ and the elements of $P$ as follows: It visits each node $S^{\prime}$ of $\Delta$ in post-order (i.e., before visiting a node it first visits its children). For each subtree $\Delta\left(S^{\prime}\right)$ of $\Delta$, it calls DMeEtApP $\left(\Delta\left(S^{\prime}\right), c\right)$ for every $c \in P$ in increasing order with respect to the order of $L$ : I.e., before calling $\operatorname{DMeEtApp}\left(\Delta\left(S^{\prime}\right), c\right)$ it calls first DMeEtApP $\left(\Delta\left(S^{\prime}\right), c^{\prime}\right)$ for each $c^{\prime} \in(P \cap \downarrow c) \backslash\{c\}$. The correctness of the call $\operatorname{DMeEt}(L, S, P)$ follows from that of $\operatorname{DMeEtApp}(\Delta, c)$. 


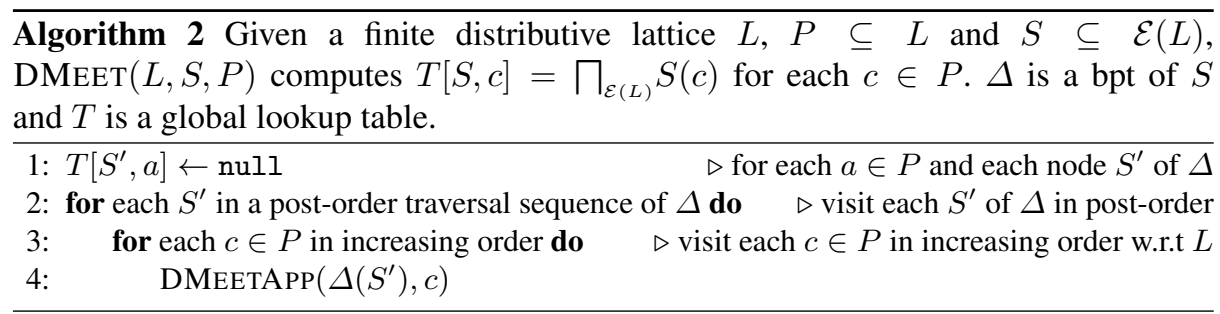

Complexity for Distributive Lattices. Assume that $L$ is a distributive lattice of size $n$ and that $S$ is a subset of $\mathcal{E}(L)$ of size $m$. The above-mentioned traversals of $\Delta$ and $P$ ensure that the assumption in Obs 3 is satisfied by each call of the form $\operatorname{DMeETApP}\left(\Delta\left(S^{\prime}\right), c\right)$ performed during the execution of $\operatorname{DMEET}(L, S)$. From Prop 6 we know that the number of iterations of the outer for is $2 m-1$. Clearly $|\downarrow c|$ and $|P|$ are both in $O(n)$. Thus, given $S^{\prime}$ we conclude from Obs 3 that the total number of operations from all calls of the form DMeEtApp $\left(\Delta\left(S^{\prime}\right), c\right)$, executed in the inner for, is in $O\left(n^{2}\right)$. The worst-case time complexity of DMeET $(L, S, L)$ is then in $O\left(m n^{2}\right)$.

Complexity for Powerset Lattices. Assume now that $L$ is a powerset lattice. We can compute $\prod_{\mathcal{E}(L)} S$ in a much lower worst-case time complexity as follows: First call $\operatorname{DMeEt}(L, S, P)$ where $P=J(L) \cup\{\perp\}$ and $J(L)$ is the set of join-irreducible elements (i.e., the singleton sets in this case) of $L$. Since $|J(L)|=\log _{2} n$ and $|\downarrow c|=2$ for every $c \in J(L)$, $\operatorname{DMeEt}(L, S, P)$ can be performed in $O(m \log n)$. This produces $T[S, c]=\left(\prod_{\mathcal{E}(L)} S\right)(c)$ for each $c \in P \subseteq L$. The computation of $T[S, e]=$ $\left(\Pi_{\mathcal{E}(L)} S\right)(e)$ for each $e \in L \backslash P$ can be performed in $O(n)$. This can be achieved by visiting each $e \in L \backslash P$ in increasing order and setting $T[S, e]=T[S, a] \sqcup T[S, b]$ for some $a, b \in \downarrow e \backslash\{e\}$ such that $e=a \sqcup b$. Since $e \notin P$ there must be $a$ and $b$ satisfying the above conditions. The total cost of computing $\prod_{\mathcal{E}(L)} S$ is therefore in $O(n+m \log n)$.

\subsection{Algorithms for Arbitrary Lattices}

The previous algorithm may fail to produce the $\prod_{\mathcal{E}(L)} S$ for non-distributive finite lattices. Nonetheless, for any arbitrary finite lattice $L, \prod_{\mathcal{E}(L)} S$ can be computed by successive approximations, starting with some self-map known to be smaller than each $f \in S$ and greater than $\prod_{\mathcal{E}(L)} S$. Assume a self-map $\sigma: L \rightarrow L$ such that $\sigma \sqsupseteq \prod_{\mathcal{E}(L)} S$ and, for all $f \in S, \sigma \sqsubseteq f$. A good starting point is $\sigma(u)=\prod\{f(u) \mid f \in S\}$, for all $u \in L$. By definition of $\sqcap, \sigma(u)$ is the biggest function under all functions in $S$, hence $\sigma \sqsupseteq \prod_{\mathcal{E}(L)} S$. The program GMEET in Algorithm 3 computes decreasing upper bounds of $\prod_{\mathcal{E}(L)} S$ by correcting $\sigma$ values not conforming to the following join-endomorphism property: $\sigma(u) \sqcup \sigma(v)=\sigma(u \sqcup v)$. The correction decreases $\sigma$ and maintains the invariant $\sigma \sqsupseteq \prod_{\mathcal{E}(L)} S$, as stated in Thm 4

Theorem 4. Let $L$ be a finite lattice, $u, v \in L, \sigma: L \rightarrow L$ and $S \subseteq \mathcal{E}(L)$. Assume $\sigma \sqsupseteq \prod_{\mathcal{E}(L)} S$ holds, and consider the following updates:

1. when $\sigma(u) \sqcup \sigma(v) \sqsubset \sigma(u \sqcup v)$, assign $\sigma(u \sqcup v) \leftarrow \sigma(u) \sqcup \sigma(v)$ 
2. when $\sigma(u) \sqcup \sigma(v) \nsubseteq \sigma(u \sqcup v)$, assign $\sigma(u) \leftarrow \sigma(u) \sqcap \sigma(u \sqcup v)$ and also $\sigma(v) \leftarrow$ $\sigma(v) \sqcap \sigma(u \sqcup v)$

Let $\sigma^{\prime}$ be the function resulting after the update. Then, (1) $\sigma^{\prime} \sqsubset \sigma$ and (2) $\sigma^{\prime} \sqsupseteq \prod_{\mathcal{E}(L)} S$.

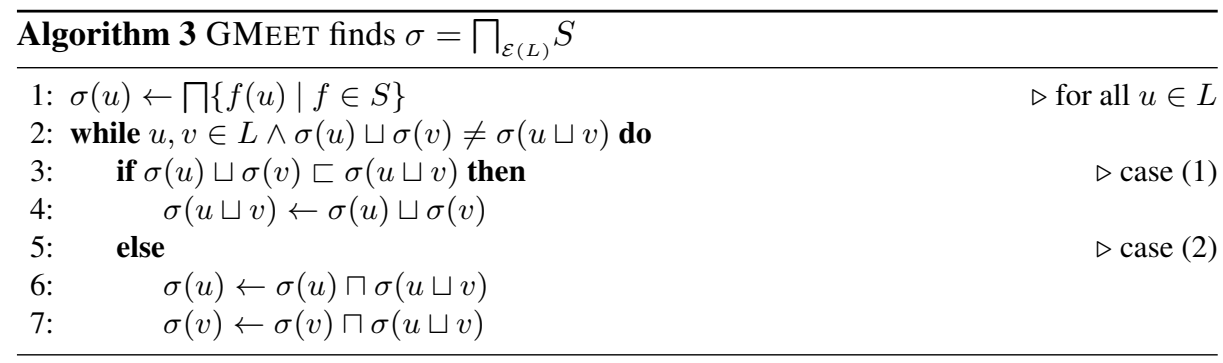

The procedure (see Algo 3 loops through pairs $u, v \in L$ while there is some pair satisfying cases (1) or (2) above for the current $\sigma$. When there is, it updates $\sigma$ as mentioned in Thm 4. At the end of the loop all pairs $u, v \in L$ satisfy the join preservation property. By the invariant mentioned in the theorem, this means $\sigma=\prod_{\mathcal{E}(L)} S$.

As for the previous algorithms in this paper the worst-time time complexity will be expressed in terms of the binary lattice operations performed during execution. Assume a fixed set $S$ of size $m$. The complexity of the initialization (Line 1) of GMEET is $O(n m)$ with $n=|L|$. The value of $\sigma$ for a given $w \in L$ can be updated (decreased) at most $n$ times. Thus, there are at most $n^{2}$ updates of $\sigma$ for all values of $L$. Finding a $w=u \sqcup v$ where $\sigma(w)$ needs an update because $\sigma(u) \sqcup \sigma(v) \neq \sigma(u \sqcup v)$ (test of the loop, Line 2) takes $O\left(n^{2}\right)$. Hence, the worst time complexity of the loop is in $O\left(n^{4}\right)$.

The program GMEET+ in Algo 4 uses appropriate data structures to reduce significantly the time complexity of the algorithm. Essentially, different sets are used to keep track of properties of $(u, v)$ lattice pairs with respect to the current $\sigma$. We have a support (correct) pairs set $\operatorname{Sup}_{w}=\{(u, v) \mid w=u \sqcup v \wedge \sigma(u) \sqcup \sigma(v)=\sigma(w)\}$. We also have a conflicts set $\operatorname{Con}_{w}=\{(u, v) \mid w=u \sqcup v \wedge \sigma(u) \sqcup \sigma(v) \sqsubset \sigma(w)\}$ and failures set $\mathrm{Fail}_{w}=\{(u, v) \mid w=u \sqcup v \wedge \sigma(u) \sqcup \sigma(v) \nsubseteq \sigma(w)\}$.

Algorithm 4 updates $\sigma$ as mentioned in Thm 4 and so maintains the invariant $\sigma \sqsupseteq$ $\prod_{\mathcal{E}(L)} S$. An additional invariant is that, for all $w$, sets $\operatorname{Sup}_{w}, \operatorname{Con}_{w}, \mathrm{Fail}_{w}$ are pairwise disjoint. When the outer loop finishes sets $\mathrm{Con}_{w}$ and $\mathrm{Fail}_{w}$ are empty (for all $w$ ) and thus every $(u, v)$ belongs to $\operatorname{Sup}_{u \sqcup v}$, i.e. the resulting $\sigma=\prod_{\mathcal{E}(L)} S$.

Auxiliary procedure CHECKSUPPORTS $(u)$ identifies all pairs of the form $(u, x) \in$ $\operatorname{Sup}_{u \sqcup x}$ that may no longer satisfy the join-endomorphism property $\sigma(u) \sqcup \sigma(x)=$ $\sigma(u \sqcup x)$ because of an update to $\sigma(u)$. When this happens, it adds $(u, x)$ to the appropriate Con, or Fail set. The time complexity of the algorithm depends on the set operations computed for each $w \in L$ chosen, either in the conflicts $\operatorname{Con}_{w}$ set or in the failures $\mathrm{Fail}_{w}$ set. When a $w$ is selected (for some $(u, v)$ such that $u \sqcup v=w$ ) the following holds: (1) at least one of $\sigma(w), \sigma(u), \sigma(v)$ is decreased, (2) some fix $k$ number of elements are removed from or added to a set, (3) a union of two disjoint sets is computed, and (4) new support sets of $w, u$ or $v$ are calculated. 


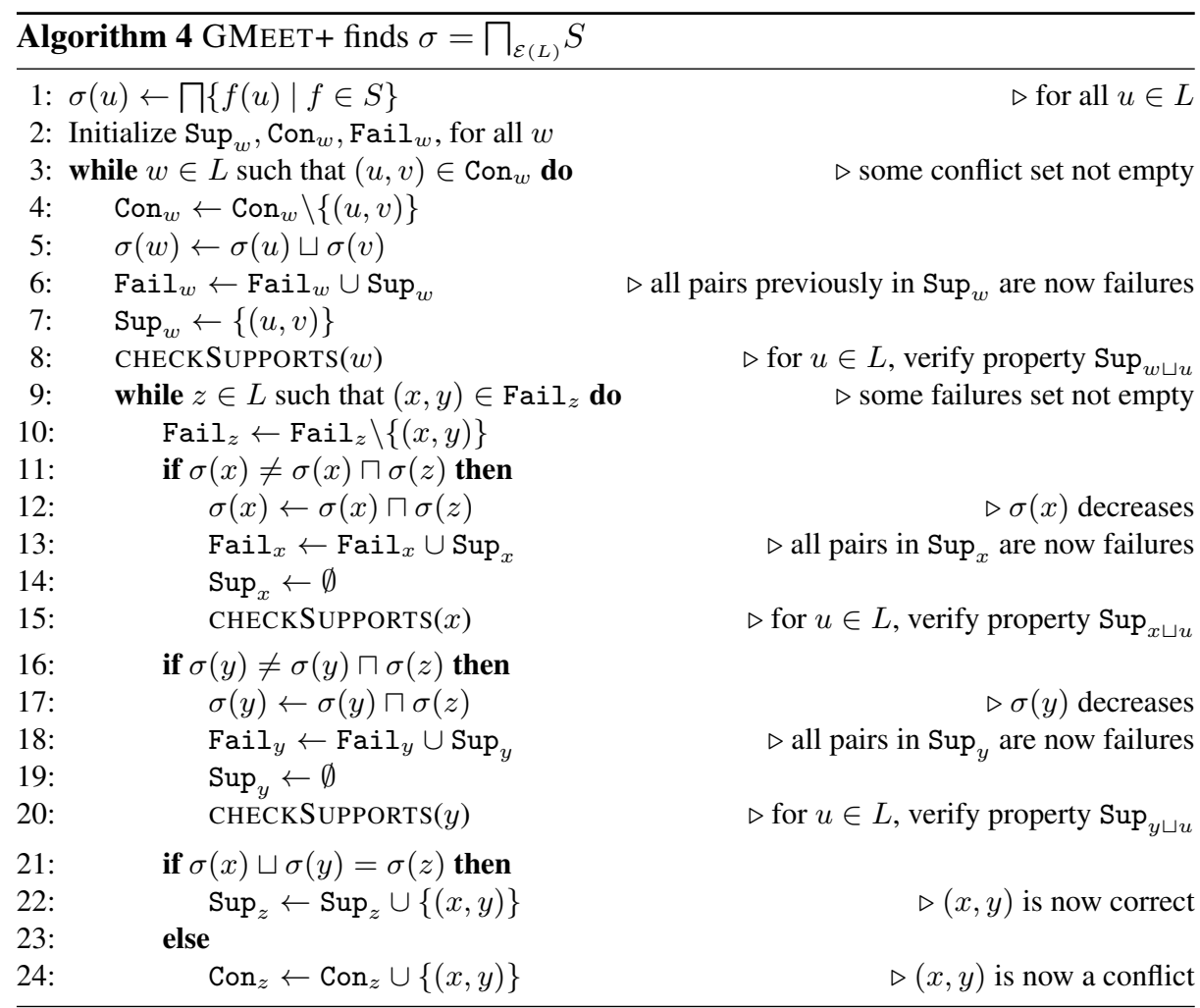

With an appropriate implementation, operations (1)-(2) take $O(1)$, and also operation (3), since sets are disjoint. Operation (4) clearly takes $O(n)$. In each loop of the (outer or inner) cycles of the algorithm, at least one $\sigma$ reduction is computed. Furthermore, for each reduction of $\sigma, O(n)$ operations are performed. The maximum possible number of $\sigma(w)$ reductions, for a given $w$, is equal to the length $d$ of the longest strictly decreasing chain in the lattice. The total number of possible $\sigma$ reductions is thus equal to $n d$. The total number of operations of the algorithm is then $O\left(n^{2} d\right)$. In general, $d$ could be (at most) equal to $n$, therefore, after initialization, worst case complexity is $O\left(n^{3}\right)$. The initialization (Lines 1-2) takes $O(n m)+O\left(n^{2}\right)$, where $m=|S|$. Worst time complexity is thus $O\left(m n+n^{3}\right)$. For powerset lattices, $d=\log _{2} n$, thus worst time complexity in this case is $O\left(m n+n^{2} \log _{2} n\right)$.

\subsection{Experimental Results and Small Example}

Here we present some experimental results showing the execution time of the proposed algorithms. We also discuss a small example with join-endomorphisms representing dilation operators from Mathematical Morphology [2]. We use the algorithms presented above to compute the greatest dilation below a given set of dilations and illustrate its result for a simple image. 

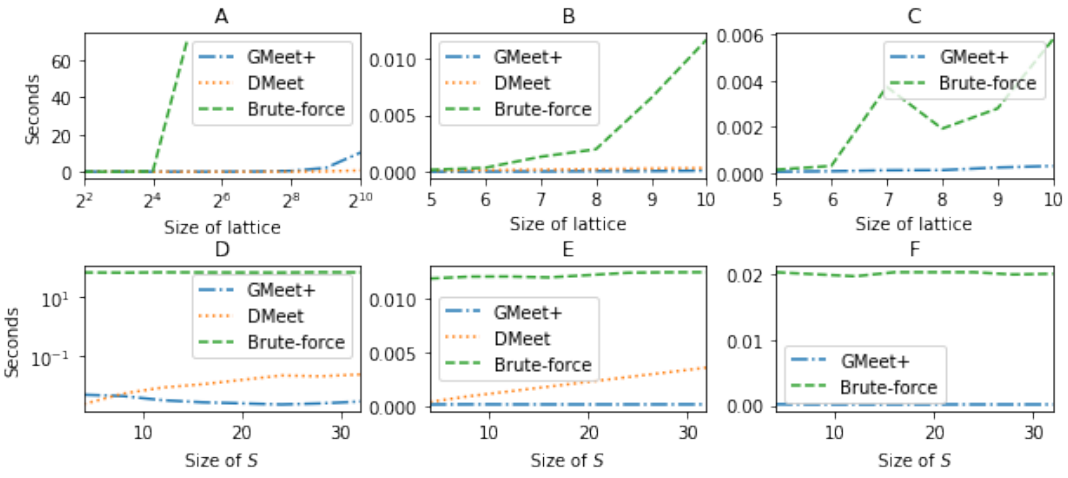

Fig. 2: Average performance time of GMEET+, DMEET and BRUTE-ForCE. Plots A and D use $\mathbf{2}^{n}$ lattices, B and $\mathrm{E}$ distributive lattices, and $\mathrm{C}$ and $\mathrm{F}$ arbitrary (possibly non-distributive) lattices. Plots A-C have a fixed number of join-endomorphisms and plots D-F have a fixed lattice size.

Consider Figure 2 In plots 2 A-C, the horizontal axis is the size of the lattice. In plots 2.D-F, the horizontal axis is the size of $S$. Curves in images 2 A-C plot, for each algorithm, the average execution time of 100 runs (10 for 2 A) with random sets $S \subseteq$ $\mathcal{E}(L)$ of size 4 . Images $2 \mathrm{D}-\mathrm{F}$, show the mean execution time of each algorithm for 100 runs (10 for 2D) varying the number of join-endomorphisms $(|S|=4 i, 1 \leq i \leq$ 8). The lattice size is fixed: $|L|=10$ for $2 \mathrm{E}$ and $2 \mathrm{~F}$, and $|L|=2^{5}$ for $2 \mathrm{D}$. In all cases the lattices were randomly generated, and the parameters selected to showcase the difference between each algorithm with a sensible overall execution time. For a given lattice $L$ and $S \subseteq \mathcal{E}(L)$, the brute-force algorithm explores the whole space $\mathcal{E}(L)$ to find all the join-endomorphism below each element of $S$ and then computes the greatest of them. In particular, the measured spike in plot $2 \mathrm{C}$ corresponds to the random lattice of seven elements with the size of $\mathcal{E}(L)$ being bigger than in the other experiments in the same figure. In our experiments we observed that for a fixed $S$, as the size of the lattice increases, DMEET outperforms GMEET+. This is noticeable in lattices $\mathbf{2}^{n}$ (see 2.A). Similarly, for a fixed lattice, as the size of $S$ increases GMEET+ outperforms DMEET. GMEET+ performance can actually improve with a higher number of joinendomorphisms (see 2D) since the initial $\sigma$ is usually smaller in this case.

To illustrate some performance gains, Table 2 shows the mean execution time of the algorithms discussed in this paper. We include $A_{1}$ and $A_{2}$, the algorithms outlined just after Lemma 1 and Proposition 5

An MM Example. Mathematical morphology (MM) is a theory, based on topological, lattice-theoretical and geometric concepts, for the analysis of geometric structures. Its algebraic framework comprises [2]14]17], among others, complete lattices together with certain kinds of morphisms, such as dilations, defined as join-endomorphisms [14]. Our results give bounds about the number of all dilations over certain specific finite lattices and also efficient algorithms to compute their infima.

A typical application of MM is image processing. Consider the space $G=\mathbb{Z}^{2}$. A dilation [2] by $s_{i} \subseteq \mathcal{P}(G)$ is a function $\delta_{s_{i}}: \mathcal{P}(G) \rightarrow \mathcal{P}(G)$ such that $\delta_{s_{i}}(X)=$ 


\begin{tabular}{rrrrrr}
\hline Size & $A_{1}$ & $A_{2}$ & GMEET & GMEET+ & DMEET \\
\hline 16 & 2.01 & 0.958 & 0.00360 & 0.000603 & 0.000632 \\
32 & 64.6 & 25.3 & 0.0633 & 0.00343 & 0.00181 \\
64 & 1901 & 600 & 0.948 & 0.0154 & 0.00542 \\
128 & $>600$ & $>600$ & 15.4 & 0.0860 & 0.0160 \\
256 & $>600$ & $>600$ & 252 & 0.361 & 0.0483 \\
512 & $>600$ & $>600$ & $>600$ & 2.01 & 0.166 \\
1024 & $>600$ & $>600$ & $>600$ & 10.7 & 0.547 \\
\hline
\end{tabular}

Table 2: Average time in seconds over powerset lattices with $|S|=4$

$\left\{x+e \mid x \in X\right.$ and $\left.e \in s_{i}\right\}$. The dilation $\delta_{s_{i}}(X)$ describes the interaction of an image $X$ with the structuring element $s_{i}$. Intuitively, the dilation of $X$ by $s_{i}$ is the result of superimpose $s_{i}$ on every activated pixel of $X$, with the center of $s_{i}$ aligned with the corresponding pixel of $X$. Then, each pixel of every superimposed $s_{i}$ is included in $\delta_{s_{i}}(X)$.

Let $L$ be the powerset lattice for some finite set $D \subseteq G$. It turns out that the dilation $\prod_{\mathcal{E}(L)} S$ corresponds to the intersection of the structuring elements of the corresponding dilations in $S$. Fig 3 illustrates $\prod_{\mathcal{E}(L)} S$ for the two given dilations $\delta_{s_{1}}(I)$ and $\delta_{s_{2}}(I)$ with structuring elements $s_{1}$ and $s_{2}$ over the given image $I$.

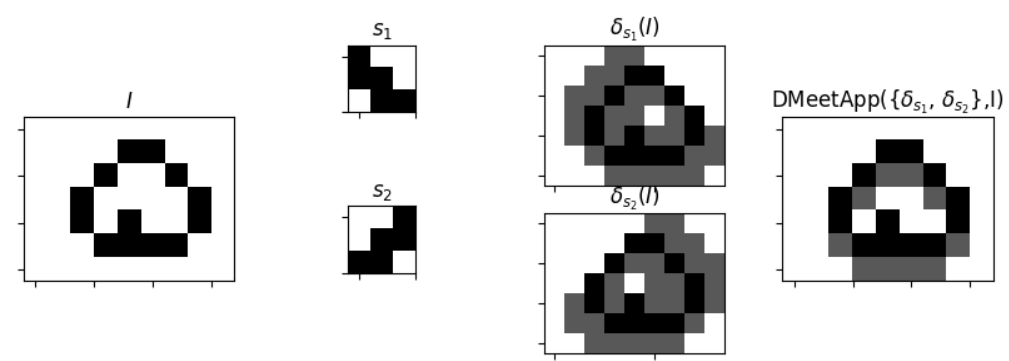

Fig. 3: Binary image $I$ (on the left). Dilations $\delta_{s_{1}}, \delta_{s_{2}}$ for structuring elements $s_{1}, s_{2}$. On the right $\left(\prod_{\mathcal{E}(L)}\left\{\delta_{s_{1}}, \delta_{s_{2}}\right\}\right)(I)$. New elements of the image after each operation in grey and black.

\section{Conclusions and Related Work}

We have shown that given a lattice $L$ of size $n$ and a set $S \subseteq \mathcal{E}(L)$ of size $m, \prod_{\mathcal{E}(L)} S$ can be computed in the worst-case in $O(n+m \log n)$ binary lattice operations for powerset lattices, $O\left(m n^{2}\right)$ for lattices of sets, and $O\left(n m+n^{3}\right)$ for arbitrary lattices. We illustrated the experimental performance of our algorithms and a small example from mathematical morphology.

In [10] a bit-vector representation of a lattice is discussed. This work gives algorithms of logarithmic (in the size of the lattice) complexity for join and meet opera- 
tions. These results count bit-vector operations. From [1] we know that $\mathcal{E}(L)$ is isomorphic to the downset of $\left(P \times P^{o p}\right)$, where $P$ is the set of join-prime elements of $L$, and that this, in turn, is isomorphic to the set of order-preserving functions from $\left(P \times P^{o p}\right)$ to 2 . Therefore, for the problem of computing $\prod_{\mathcal{E}(L)} S$, we get bounds $O\left(m \log _{2}\left(2^{\left(n^{2}\right)}\right)=O\left(m n^{2}\right)\right.$ for set lattices and $O\left(m\left(\log _{2} n\right)^{2}\right)$ for powerset lattices where $n=|L|$ and $m=|S|$. This, however, assumes a bit-vector representation of a lattice isomorphic to $\mathcal{E}(L)$. Computing this representation takes time and space proportional to the size of $\mathcal{E}(L)$ [10] which could be exponential as stated in the present paper. Notice that in our algorithms the input lattice is $L$ instead of $\mathcal{E}(L)$.

We have stated the cardinality of the set of join-endomorphisms $\mathcal{E}(L)$ for significant families of lattices. To the best of our knowledge we are the first to establish the cardinality $(n+1)^{2}+n ! \mathcal{L}_{n}(-1)$ for the lattice $\mathbf{M}_{n}$. The cardinalities $n^{\log _{2} n}$ for power sets (boolean algebras) and $\left(\begin{array}{c}2 n \\ n\end{array}\right)$ for linear orders can also found in the lattice literature [1111[16]. We presented our original proofs of these statements.

The lattice $\mathcal{E}(L)$ have been studied in [7]. The authors showed that a finite lattice $L$ is distributive iff $\mathcal{E}(L)$ is distributive. A lower bound of $2^{2 n / 3}$ for the number of monotonic self-maps of any finite poset $L$ is given in [4]. Nevertheless to the best of our knowledge, no other authors have studied the problem of determining the size $\mathcal{E}(L)$ nor algorithms for computing $\prod_{\mathcal{E}(L)} S$. We believe that these problems are important, as argued in the Introduction, algebraic structures consisting of a lattice and joinendomorphisms are very common in mathematics and computer science. In fact, our interest in this subject arose in the algebraic setting of spatial and epistemic constraint systems [9] where continuous join-endomorphisms, called space functions, represent knowledge and the infima of endomorphisms correspond to distributed knowledge. We showed in [9] that distributed knowledge can be computed in $O\left(m n^{1+\log _{2}(m)}\right)$ for distributive lattices and $O\left(n^{4}\right)$ in general. In this paper we have provided much lower complexity orders for computing infima of join-endomorphisms. Furthermore [9] does not provide the exact cardinality of the set of space function of a given lattice.

As future work we plan to explore in detail the applications of our work in mathematical morphology and computer music [15]. Furthermore, in the same spirit of [12] we have developed algorithms to generate distributive and arbitrary lattices. In our experiments, we observed that for every lattice $L$ of size $n$ we generated, $n^{\log _{2} n} \leq$ $|\mathcal{E}(L)| \leq(n+1)^{2}+n ! \mathcal{L}_{n}(-1)$ and if the generated lattice was distributive, $n^{\log _{2} n} \leq$ $|\mathcal{E}(L)| \leq\left(\begin{array}{c}2 n \\ n\end{array}\right)$. We plan to establish if these inequalities hold for every finite lattice.

Acknowledgments. We are indebted to the anonymous referees and editors of RAMICS 2020 for helping us improve one of the complexity bound, some proofs, and the overall quality of the paper.

\section{References}

1. Birkhoff, G.: Lattice Theory. No. v. 25,pt. 2 in American Mathematical Society colloquium publications, American Mathematical Society (1967)

2. Bloch, I., Heijmans, H., Ronse, C.: Mathematical morphology. In: Aiello, M., PrattHartmann, I., Van Benthem, J. (eds.) Handbook of Spatial Logics. pp. 857-944. Springer Netherlands (2007) 
3. Davey, B.A., Priestley, H.A.: Introduction to lattices and order. Cambridge university press, 2nd edn. (2002)

4. Duffus, D., Rodl, V., Sands, B., Woodrow, R.: Enumeration of order preserving maps. Order 9(1), 15-29 (1992)

5. Feller, W.: An introduction to probability theory and its applications. Wiley series in probability and mathematical statistics: Probability and mathematical statistics, Wiley (1971)

6. Gierz, G., Hofmann, K.H., Keimel, K., Lawson, J.D., Mislove, M., Scott, D.S.: Continuous lattices and domains. Cambridge University Press (2003)

7. Gr'atzer, G., Schmidt, E.: On the lattice of all join-endomorphisms of a lattice. Proceedings of The American Mathematical Society - PROC AMER MATH SOC 9, 722-722 (1958)

8. Guzmán, M., Haar, S., Perchy, S., Rueda, C., Valencia, F.D.: Belief, knowledge, lies and other utterances in an algebra for space and extrusion. J. Log. Algebr. Meth. Program. 86(1), 107-133 (2017)

9. Guzmán, M., Knight, S., Quintero, S., Ramírez, S., Rueda, C., Valencia, F.D.: Reasoning about Distributed Knowledge of Groups with Infinitely Many Agents. In: CONCUR 2019 30th International Conference on Concurrency Theory. vol. 29, pp. 1-29 (2019)

10. Habib, M., Nourine, L.: Tree structure for distributive lattices and its applications. Theoretical Computer Science 165(2), 391 - 405 (1996)

11. Jipsen, P.: Relation algebras, idempotent semirings and generalized bunched implication algebras. In: Relational and Algebraic Methods in Computer Science. pp. 144-158. Springer International Publishing (2017)

12. Jipsen, P., Lawless, N.: Generating all finite modular lattices of a given size. Algebra universalis 74(3), 253-264 (2015)

13. Knight, S., Palamidessi, C., Panangaden, P., Valencia, F.D.: Spatial and Epistemic Modalities in Constraint-Based Process Calculi. In: 23rd International Conference on Concurrency Theory. Lecture Notes in Computer Science, vol. 7454, pp. 317-332. Springer (2012)

14. Ronse, C.: Why mathematical morphology needs complete lattices. Signal Processing 21(2), 129-154 (1990)

15. Rueda, C., Valencia, F.: On validity in modelization of musical problems by ccp. Soft Computing 8(9), 641-648 (2004)

16. Santocanale, L.: On Discrete Idempotent Paths. In: Combinatorics on Words. vol. 11682, pp. 312-325. Springer (2019)

17. Stell, J.: Why mathematical morphology needs quantales. In: Wilkinson, M., Roerdink, J. (eds.) International Symposium on Mathematical Morphology, ISMM09. pp. 13-16. Institute for Mathematics and Computing Science, University of Groningen (2009) 


\section{A Proofs}

\section{A.1 Proof of Lemma 1}

Let $L$ be a finite distributive lattice and $S=\left\{f_{i}\right\}_{i \in I} \subseteq \mathcal{E}(L)$. Then $\prod_{\mathcal{E}(L)} S=$ $\delta_{S}$ where $\delta_{S}(c) \stackrel{\text { def }}{=} \prod_{L}\left\{\bigsqcup_{i \in I} f_{i}\left(a_{i}\right) \mid\left(a_{i}\right)_{i \in I} \in L^{I}\right.$ and $\left.\bigsqcup_{i \in I} a_{i} \sqsupseteq c\right\}$.

Proof. Recall that $\prod_{\mathcal{E}(L)} S=\max \left\{h \in \mathcal{E}(L) \mid h \sqsubseteq_{\mathcal{E}} g\right.$ for all $\left.g \in S\right\}$ and let us define $\Gamma=\left\{\bigsqcup_{i \in I} f_{i}\left(a_{i}\right) \mid\left(a_{i}\right)_{i \in I} \in L^{I}\right.$ and $\left.\bigsqcup_{i \in I} a_{i} \sqsupseteq c\right\}$. We prove (1) $\prod_{\mathcal{E}(L)} S \sqsubseteq \mathcal{E} \delta_{S}$ and (2) $\delta_{S} \sqsubseteq \mathcal{E} \prod_{\mathcal{E}(L)} S$.

1. $\prod_{\mathcal{E}(L)} S \sqsubseteq \mathcal{E} \delta_{S}$.

Let $c \in L$ and $\left(a_{i}\right)_{i \in I} \in L^{I}$ be an arbitrary tuple such that $\bigsqcup_{i \in I} a_{i} \sqsupseteq c$. Notice that $\bigsqcup_{i \in I}\left(\prod_{\mathcal{E}(L)} S\right)\left(a_{i}\right) \sqsubseteq \bigsqcup_{i \in I} f_{i}\left(a_{i}\right)$. Since $\prod_{\mathcal{E}(L)} S$ is a join-endomorphism of $L$ and monotonic, we know that $\bigsqcup_{i \in I}\left(\prod_{\mathcal{E}(L)} S\right)\left(a_{i}\right)=\left(\prod_{\mathcal{E}(L)} S\right)\left(\bigsqcup_{i \in I} a_{i}\right) \sqsupseteq$ $\left(\prod_{\mathcal{E}(L)} S\right)(c)$. Thus $\left(\prod_{\mathcal{E}(L)} S\right)(c) \sqsubseteq \bigsqcup_{i \in I} f_{i}\left(a_{i}\right)$, i.e., $\left(\prod_{\mathcal{E}(L)} S\right)(c)$ is a lower bound of $\Gamma$. Then for every $c \in L,\left(\prod_{\mathcal{E}(L)} S\right)(c) \sqsubseteq \delta_{S}(c)$. Therefore $\prod_{\mathcal{E}(L)} S \sqsubseteq \mathcal{E} \delta_{S}$.

2. $\delta_{S} \sqsubseteq \mathcal{E} \prod_{\mathcal{E}(L)} S$.

We prove (a) $\delta_{S} \in \mathcal{E}(L)$ and (b) $\delta_{S} \sqsubseteq \mathcal{E} f_{i}$ for every $f_{i} \in S$.

(a) Prove that $\delta_{S} \sqsubseteq_{\mathcal{E}} f_{i}$, for every $f_{i} \in S$.

Let $c \in L$. From definition of $\delta_{S}$, for every $i \in I$, the element $f_{i}(c)=f_{i}(c) \sqcup$ $\bigsqcup_{j \in I \backslash\{i\}} f_{j}(\perp) \in \Gamma$. Then for every $c \in L, \delta_{S}(c) \sqsubseteq f_{i}(c)$. Therefore for every $f_{i} \in S, \delta_{S} \sqsubseteq \mathcal{E} f_{i}$.

(b) $\delta_{S} \in \mathcal{E}(L)$.

We show that for any $H \subseteq L, \delta_{S}(\bigsqcup H)=\bigsqcup\left\{\delta_{S}(e) \mid\right.$ for every $\left.e \in H\right\}$. Since $H$ is finite, it suffices to show that our claim holds for $H=\emptyset$ and $H=\{c, d\}$.

Assume $H=\emptyset$. One can verify that $\delta_{S}(\perp)=\perp$.

Assume $H=\{c, d\}$. Firstly, we prove that $\delta_{S}$ is monotonic. Suppose $c \sqsupseteq d$. For any $\left(a_{i}\right)_{i \in I} \in L^{I}$ such that $\bigsqcup_{i \in I} a_{i} \sqsupseteq c$, we have $\bigsqcup_{i \in I} a_{i} \sqsupseteq d$. Therefore, $\left\{\bigsqcup_{i \in I} f_{i}\left(a_{i}\right) \mid \bigsqcup_{i \in I} a_{i} \sqsupseteq c\right\} \subseteq\left\{\bigsqcup_{i \in I} f_{i}\left(a_{i}\right) \mid \bigsqcup_{i \in I} a_{i} \sqsupseteq d\right\}$ which implies $\delta_{S}(c) \sqsupseteq \delta_{S}(d)$.

By monotonicity of $\delta_{S}$, we know $\delta_{S}(c \sqcup d) \sqsupseteq \delta_{S}(c) \sqcup \delta_{S}(d)$. The other direction follows from the derivation below: 


$$
\begin{aligned}
& \delta_{S}(c) \sqcup \delta_{S}(d) \\
= & \left\langle\text { Definition of } \delta_{S}(d)\right\rangle \\
& \delta_{S}(c) \sqcup \prod_{L}\left\{\bigsqcup_{i \in I} f_{i}\left(b_{i}\right) \mid\left(b_{i}\right)_{i \in I} \in L^{I} \text { and } \bigsqcup_{i \in I} b_{i} \sqsupseteq d\right\} \\
= & \langle\sqcup \text { distributes over } \sqcap\rangle \\
& \prod_{L}\left\{\delta_{S}(c) \sqcup \bigsqcup_{i \in I} f_{i}\left(b_{i}\right) \mid\left(b_{i}\right)_{i \in I} \in L^{I} \text { and } \bigsqcup_{i \in I} b_{i} \sqsupseteq d\right\} \\
= & \left\langle\text { Definition of } \delta_{S}(c)\right\rangle \\
& \prod_{L}\left\{\prod_{L}\left\{\bigsqcup_{i \in I} f_{i}\left(a_{i}\right) \mid\left(a_{i}\right)_{i \in I} \in L^{I} \text { and } \bigsqcup_{i \in I} a_{i} \sqsupseteq c\right\} \sqcup \bigsqcup_{i \in I} f_{i}\left(b_{i}\right) \mid\left(b_{i}\right)_{i \in I} \in L^{I} \text { and } \bigsqcup_{i \in I} b_{i} \sqsupseteq d\right\} \\
= & \langle\sqcup \text { distributes over } \sqcap\rangle \\
& \prod_{L}\left\{\prod_{L}\left\{\bigsqcup_{i \in I} f_{i}\left(a_{i}\right) \sqcup \bigsqcup_{i \in I} f_{i}\left(b_{i}\right) \mid\left(a_{i}\right)_{i \in I} \in L^{I} \text { and } \bigsqcup_{i \in I} a_{i} \sqsupseteq c\right\} \mid\left(b_{i}\right)_{i \in I} \in L^{I} \text { and } \bigsqcup_{i \in I} b_{i} \sqsupseteq d\right\} \\
= & \langle\text { Associativity of } \sqcap\rangle \\
& \prod_{L}\left\{\bigsqcup_{i \in I}\left(f_{i}\left(a_{i}\right) \sqcup f_{i}\left(b_{i}\right)\right) \mid\left(a_{i}\right)_{i \in I},\left(b_{i}\right)_{i \in I} \in L^{I} \text { and } \bigsqcup_{i \in I} a_{i} \sqsupseteq c \text { and } \bigsqcup_{i \in I} b_{i} \sqsupseteq d\right\} \\
\sqsupseteq & \left\langle x \sqsupseteq y \text { and } w \sqsupseteq z \text { implies } x \sqcup w \sqsupseteq y \sqcup z ; c_{i}=a_{i} \sqcup b_{i} ; f_{i}\left(c_{i}\right)=f_{i}\left(a_{i} \sqcup b_{i}\right)\right\rangle \\
& \prod_{L}\left\{\bigsqcup_{i \in I} f_{i}\left(c_{i}\right) \mid\left(c_{i}\right)_{i \in I} \in L^{I} \text { and } \bigsqcup_{i \in I} c_{i} \sqsupseteq c \sqcup d\right\} \\
= & \left\langle\text { Definition of } \delta_{S}(c \sqcup d)\right\rangle \\
& \delta_{S}(c \sqcup d)
\end{aligned}
$$

Thus we conclude $\delta_{S} \in \mathcal{E}(L)$.

From items (a) and (b), $\delta_{S} \sqsubseteq \mathcal{E} \prod_{\mathcal{E}(L)} S$ holds.

We conclude $\prod_{\mathcal{E}(L)} S=\delta_{S}$.

\section{A.2 Proof of Corollary 2 .}

We wish to prove that $|A|=\mathcal{R}_{n}(1)$ where $A=\left\{f \in \mathcal{E}\left(\mathbf{M}_{n}\right) \mid f\right.$ is non-reducing in $\left.\mathbf{M}_{n}\right\}$. Let $\mathcal{F}=\bigcup_{i=1}^{4} \mathcal{F}_{i}$ where the mutually exclusive $\mathcal{F}_{i}$ 's are defined in Table 1 In the proof of Theorem 1 we show that $\mathcal{E}\left(\mathbf{M}_{n}\right)=\mathcal{F}$ and that $\mathcal{R}_{n}(1)=\left|\mathcal{F}_{4}\right|$. Notice that every function in $\mathcal{F}_{4}$ is non-reducing and every function in $\mathcal{F} \backslash \mathcal{F}_{4}$ is not non-reducing. Hence $A=\mathcal{F}_{4}$, thus $|A|=\mathcal{R}_{n}(1)$.

\section{A.3 Proof of Proposition 5}

Let $(L, \sqsubseteq)$ be a finite distributive lattice and $S=\left\{f_{i}\right\}_{i \in I} \subseteq \mathcal{E}(L)$. Let $S_{1}, S_{2} \subseteq \mathcal{E}(L)$ be such that $S=S_{1} \cup S_{2}$. Then

$$
\left(\prod_{\mathcal{E}(L)} S\right)(c)=\prod_{L}\left\{\left(\prod_{\mathcal{E}(L)} S_{1}\right)(a) \sqcup\left(\prod_{\mathcal{E}(L)} S_{2}\right)(b) \mid a \sqcup b \sqsupseteq c\right\} .
$$


Proof. The proof follows using the fact that $\prod_{\mathcal{E}(L)} S \in \mathcal{E}(L)$ and Lemma 1 .

Let $a, b, c \in L$ such that $a \sqcup b \sqsubseteq c$. Let $S_{1}=\left\{f_{j}\right\}_{j \in J}$ and $S_{2}=\left\{f_{k}\right\}_{k \in K}$ such that $I=J \cup K$. From Lemma1

$$
\left(\prod_{\mathcal{E}(L)} S_{1}\right)(a)=\prod_{L}\left\{\bigsqcup_{j \in J} f_{j}\left(a_{j}\right) \mid\left(a_{j}\right)_{j \in J} \in L^{J} \text { and } \bigsqcup_{j \in J} a_{j} \sqsupseteq a\right\}
$$

and

$$
\left(\prod_{\mathcal{E}(L)} S_{2}\right)(b)=\prod_{L}\left\{\bigsqcup_{k \in K} f_{k}\left(b_{k}\right) \mid\left(b_{k}\right)_{k \in K} \in L^{K} \text { and } \bigsqcup_{k \in K} b_{k} \sqsupseteq b\right\}
$$

By distributivity of $\sqcup$ over $\sqcap,\left(\prod_{\mathcal{E}(L)} S_{1}\right)(a) \sqcup\left(\prod_{\mathcal{E}(L)} S_{2}\right)(b)$ can be written as:

$$
\prod_{L}\left\{\bigsqcup_{i \in I} f_{i}\left(c_{i}\right) \mid\left(c_{i}\right)_{i \in I} \in L^{I} \text { and } \bigsqcup_{i \in I} c_{i} \sqsupseteq a \sqcup b\right\}
$$

where $c_{i}$ is either $a_{i}, b_{i}$ or $a_{i} \sqcup b_{i}$.

$$
\begin{aligned}
& \prod_{L}\left\{\left(\prod_{\mathcal{E}(L)} S_{1}\right)(a) \sqcup\left(\prod_{\mathcal{E}(L)} S_{2}\right)(b) \mid a \sqcup b \sqsupseteq c\right\} \\
= & \left\langle\text { Construction of }\left(\prod_{\mathcal{E}(L)} S_{1}\right)(a) \sqcup\left(\prod_{\mathcal{E}(L)} S_{2}\right)(b)\right\rangle \\
& \prod_{L}\left\{\prod_{L}\left\{\bigsqcup_{i \in I} f_{i}\left(c_{i}\right) \mid\left(c_{i}\right)_{i \in I} \in L^{I} \text { and } \bigsqcup_{i \in I} c_{i} \sqsupseteq a \sqcup b\right\} \mid a \sqcup b \sqsupseteq c\right\} \\
= & \langle\text { Associativity of } \sqcap\rangle \\
& \prod_{L}\left\{\bigsqcup_{i \in I} f_{i}\left(c_{i}\right) \mid\left(c_{i}\right)_{i \in I} \in L^{I} \text { and } \bigsqcup_{i \in I} c_{i} \sqsupseteq a \sqcup b \text { and } a \sqcup b \sqsupseteq c\right\} \\
= & \langle(\sqsupseteq) \text { Transitivity of } \sqsupseteq ;(\sqsubseteq \text { Associativity of } \sqcap .\rangle \\
& \prod_{L}\left\{\bigsqcup_{i \in I} f_{i}\left(c_{i}\right) \mid\left(c_{i}\right)_{i \in I} \in L^{I} \text { and } \bigsqcup_{i \in I} c_{i} \sqsupseteq c\right\} \\
= & \langle\text { Lemma回 } \\
& \left(\prod_{\mathcal{E}(L)} S\right)(c)
\end{aligned}
$$

\section{A.4 Proof of Theorem 2}

Suppose $(L, \sqsubseteq)$ is a finite distributive lattice. Let $S_{1}, S_{2} \subseteq \mathcal{E}(L)$ be such that $S=$ $S_{1} \cup S_{2} \subseteq \mathcal{E}(L)$. Then

$$
\left(\prod_{\mathcal{E}(L)} S\right)(c)=\prod_{L}\left\{\left(\prod_{\mathcal{E}(L)} S_{1}\right)(a) \sqcup\left(\prod_{\mathcal{E}(L)} S_{2}\right)(c \backslash a) \mid a \in L \text { and } a \sqsubseteq c\right\} .
$$

Proof. Firstly, we prove that

$$
\left(\prod_{\mathcal{E}(L)} S\right)(c)=\prod_{L}\left\{\left(\prod_{\mathcal{E}(L)} S_{1}\right)(a) \sqcup\left(\prod_{\mathcal{E}(L)} S_{2}\right)(c \backslash a)\right\} .
$$


From its definition $c \backslash a$ represents the least element $e$ such that $a \sqcup e \sqsupseteq c$, i.e., $c \backslash a=$ $\prod\{e \mid a \sqcup e \sqsupseteq c\}$. Take any $b$ such that $a \sqcup b \sqsupseteq c$. Then $b \sqsupseteq c \backslash a$ and since $\prod_{\mathcal{E}(L)} S_{1}$ and $\prod_{\mathcal{E}(L)} S_{2}$ are monotonic

$$
\left(\prod_{\mathcal{E}(L)} S_{1}\right)(a) \sqcup\left(\prod_{\mathcal{E}(L)} S_{2}\right)(b) \sqsupseteq\left(\prod_{\mathcal{E}(L)} S_{1}\right)(a) \sqcup\left(\prod_{\mathcal{E}(L)} S_{2}\right)(c \backslash a) .
$$

From this it follows that

$$
\begin{aligned}
& \prod_{L}\left(C \cup\left\{\left(\prod_{\mathcal{E}(L)} S_{1}\right)(a) \sqcup\left(\prod_{\mathcal{E}(L)} S_{2}\right)(c \backslash a),\left(\prod_{\mathcal{E}(L)} S_{1}\right)(a) \sqcup\left(\prod_{\mathcal{E}(L)} S_{2}\right)(b)\right\}\right) \\
= & \prod_{L}\left(C \cup\left\{\left(\prod_{\mathcal{E}(L)} S_{1}\right)(a) \sqcup\left(\prod_{\mathcal{E}(L)} S_{2}\right)(c \backslash a)\right\}\right)
\end{aligned}
$$

for any $C \subseteq L$. This shows that $\left(\prod_{\mathcal{E}(L)} S_{1}\right)(a) \sqcup\left(\prod_{\mathcal{E}(L)} S_{2}\right)(b)$ is redundant since $\left(\prod_{\mathcal{E}(L)} S_{1}\right)(a) \sqcup\left(\prod_{\mathcal{E}(L)} S_{2}\right)(c \backslash a)$ is included in the set on the right-hand side of Equation[1]

The theorem can be seen as a simplification of Equation 1 Take any $a^{\prime} \nsubseteq c$. It suffices to find $a \sqsubseteq c$ such that

$$
\left(\prod_{\mathcal{E}(L)} S_{1}\right)\left(a^{\prime}\right) \sqcup\left(\prod_{\mathcal{E}(L)} S_{2}\right)\left(c \backslash a^{\prime}\right) \sqsupseteq\left(\prod_{\mathcal{E}(L)} S_{1}\right)(a) \sqcup\left(\prod_{\mathcal{E}(L)} S_{2}\right)(c \backslash a)
$$

since then for any $C \subseteq L$

$$
\begin{aligned}
& \prod_{L}\left(C \cup\left\{\left(\prod_{\mathcal{E}(L)} S_{1}\right)(a) \sqcup\left(\prod_{\mathcal{E}(L)} S_{2}\right)(c \backslash a),\left(\prod_{\mathcal{E}(L)} S_{1}\right)\left(a^{\prime}\right) \sqcup\left(\prod_{\mathcal{E}(L)} S_{2}\right)\left(c \backslash a^{\prime}\right)\right\}\right) \\
& =\prod_{L}\left(C \cup\left\{\left(\prod_{\mathcal{E}(L)} S_{1}\right)(a) \sqcup\left(\prod_{\mathcal{E}(L)} S_{2}\right)(c \backslash a)\right\}\right) .
\end{aligned}
$$

Since $a^{\prime} \nsubseteq c$ either (a) $a^{\prime} \sqsupset c$ or (b) $a^{\prime}$ and $c$ are incomparable w.r.t. $\sqsubseteq$, written $a^{\prime} \| c$. Suppose (a) holds. Then take $a=c$ thus $c \backslash a=$ true. By monotonicity we have $\left(\prod_{\mathcal{E}(L)} S_{1}\right)\left(a^{\prime}\right) \sqcup\left(\prod_{\mathcal{E}(L)} S_{2}\right)\left(c \backslash a^{\prime}\right) \sqsupseteq\left(\prod_{\mathcal{E}(L)} S_{1}\right)(a) \sqcup\left(\prod_{\mathcal{E}(L)} S_{2}\right)(c \backslash a)$ as wanted. Suppose that (b) $a^{\prime} \| c$ holds. Notice that $c \backslash a^{\prime} \sqsubseteq c$. Suppose that $c \backslash a^{\prime}=c$. Then we can take $a=\perp$, and thus $c \backslash a=c=c \backslash a^{\prime}$. By monotonicity we have $\left(\prod_{\mathcal{E}(L)} S_{1}\right)\left(a^{\prime}\right) \sqcup\left(\prod_{\mathcal{E}(L)} S_{2}\right)\left(c \backslash a^{\prime}\right) \sqsupseteq\left(\prod_{\mathcal{E}(L)} S_{1}\right)(a) \sqcup\left(\prod_{\mathcal{E}(L)} S_{2}\right)(c \backslash a)$ as wanted. Suppose $c \backslash a^{\prime} \sqsubset c$ holds. In this case, which is more interesting, we can build a poset $L=\left(\left\{a^{\prime} \sqcup c, a^{\prime}, c, c \backslash a^{\prime}, a^{\prime} \sqcap\left(c \backslash a^{\prime}\right)\right\}, \sqsubseteq\right)$ and verify that $L$ is a non-distributive sub-lattice of $(L, \sqsubseteq)$, isomorphic to a lattice known as $\mathbf{N}_{5}$ (see Fig. 47). But from order theory we know this cannot happen since we assumed $(L, \sqsubseteq)$ to be distributive, and distributive lattices do not have sub-lattices isomorphic to $\mathbf{N}_{5}$ ([3]).

\section{A.5 Proof of Theorem 4}

Let $L$ be a finite lattice, $u, v \in L, \sigma: L \rightarrow L$ and $S \subseteq \mathcal{E}(L)$. Assume $\sigma \sqsupseteq \prod_{\mathcal{E}(L)} S$ holds, and consider the following updates:

1. when $\sigma(u) \sqcup \sigma(v) \sqsubset \sigma(u \sqcup v)$, assign $\sigma(u \sqcup v) \leftarrow \sigma(u) \sqcup \sigma(v)$

2. when $\sigma(u) \sqcup \sigma(v) \nsubseteq \sigma(u \sqcup v)$, assign $\sigma(u) \leftarrow \sigma(u) \sqcap \sigma(u \sqcup v)$ and also $\sigma(v) \leftarrow$ $\sigma(v) \sqcap \sigma(u \sqcup v)$ 


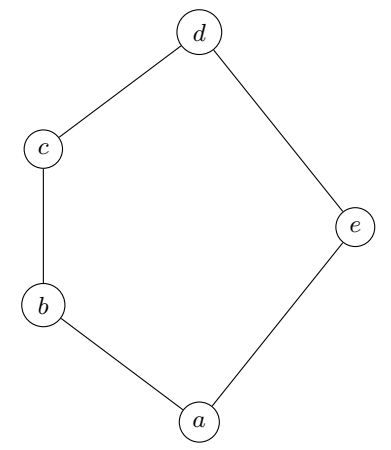

Fig. 4: Non-distributive lattice: $\mathbf{N}_{5}$.

Let $\sigma^{\prime}$ be the function resulting after the update. Then, (1) $\sigma^{\prime} \sqsubset \sigma$ and (2) $\sigma^{\prime} \sqsupseteq \prod_{\mathcal{E}(L)} S$

Proof. For update (1):

given the condition, the assignment obviously decreases $\sigma(u \sqcup v)$, so $\sigma^{\prime} \sqsubset \sigma$. For the invariant, since $\sigma \sqsupseteq \prod_{\mathcal{E}(L)} S$, then, $\sigma(u) \sqsupseteq\left(\prod_{\mathcal{E}(L)} S\right)(u)$ and $\sigma(v) \sqsupseteq\left(\prod_{\mathcal{E}(L)} S\right)(v)$, and, therefore, $\sigma^{\prime}(u \sqcup v)=\sigma(u) \sqcup \sigma(v) \sqsupseteq\left(\prod_{\mathcal{E}(L)} S\right)(u) \sqcup\left(\prod_{\mathcal{E}(L)} S\right)(v)=\left(\prod_{\mathcal{E}(L)} S\right)(u \sqcup v)$.

For update (2),

the assignments either decrease $\sigma(u)$ or $\sigma(v)$ (or both). To see why, assume the opposite, $\sigma(u)=\sigma(u) \sqcap \sigma(u \sqcup v) \rightarrow \sigma(u) \sqsubseteq \sigma(u \sqcup v)$, and also $\sigma(v)=\sigma(v) \sqcap \sigma(u \sqcup v) \rightarrow$ $\sigma(v) \sqsubseteq \sigma(u \sqcup v)$. Therefore, $\sigma(u) \sqcup \sigma(v) \sqsubseteq \sigma(u \sqcup v)$, contradicting the condition for update 2. Assignments in update 2 also preserve the invariant $\sigma \sqsupseteq \prod_{\mathcal{E}(L)} S$.

assume $\sigma(u) \sqcap \sigma(u \sqcup v) \sqsubset \sigma(u)$ (otherwise the invariant holds trivially). By the invariant hypothesis for $\sigma$ before the assignment, we have that $\sigma(u) \sqsupseteq\left(\prod_{\mathcal{E}(L)} S\right)(u)$ and $\sigma(u \sqcup v) \sqsupseteq\left(\prod_{\mathcal{E}(L)} S\right)(u \sqcup v)$. Therefore,

$$
\begin{aligned}
\sigma(u) \sqcap \sigma(u \sqcup v) & \sqsupseteq\left(\prod_{\mathcal{E}(L)} S\right)(u) \sqcap\left(\prod_{\mathcal{E}(L)} S\right)(u \sqcup v) \\
& \left.=\left(\prod_{\mathcal{E}(L)} S\right)(u) \sqcap\left(\prod_{\mathcal{E}(L)} S\right)(u) \sqcup\left(\prod_{\mathcal{E}(L)} S\right)(v)\right) \\
& =\left(\prod_{\mathcal{E}(L)} S\right)(u) \sqcup\left(\left(\prod_{\mathcal{E}(L)} S\right)(u) \sqcap\left(\prod_{\mathcal{E}(L)} S\right)(v)\right) \\
& =\left(\prod_{\mathcal{E}(L)} S\right)(u)
\end{aligned}
$$

The proof for $\sigma^{\prime}(v)$ is analogous. 